\title{
Multiscale Modeling of Hydrogen Embrittlement
}

Chapter · May 2015

DOI: 10.1002/9781119057666.ch8

CITATIONS

0

2 authors:

\section{Xu Zhang}

California State University, Northridge 70 PUBLICATIONS 767 CITATIONS

SEE PROFILE
READS

35

Gang Lu

California State University, Northridge

141 PUBLICATIONS 2,253 CITATIONS

SEE PROFILE 


\title{
CHAPTER 1
}

\section{MULTISCALE MODELING OF HYDROGEN EMBRITTLEMENT}

\author{
Xu Zhang AND Gang LU \\ Department of Physics and Astronomy, California State University Northridge, Northridge, California \\ 91330-8268, USA
}

\subsection{Introduction}

Hydrogen is a major reactant with solids as a result of its strong chemical activity, high lattice mobility, and wide occurrence as $\mathrm{H}_{2}$ molecule and a constituent of molecular gases and liquids (moisture in air, for example) [1]. As a consequence, interactions of $\mathrm{H}$ with lattice defects, such as vacancies, dislocations, grain boundaries, and cracks, are crucial in determining the influence of this impurity on the properties of solids. These interactions are the ultimate culprit of $\mathrm{H}$ embrittlement, which is one of the most important problems in materials science and engineering as almost all metals and their alloys suffer to some extent of H-induced brittleness [2]. Since the early 1960s, H-induced cracking has been responsible for many, if not most, service failures in numerous applications where components and structures come into contact with natural or technological environment - whether aqueous solution, gas, elevated temperature, or irradiation. Moreover, the problem of $\mathrm{H}$ induced cracking directly affects the safety and reliability of engineering systems such as aircraft and aerospace structures, nuclear and fossil fuel power plants, oil and gas pipelines, field equipment, chemical plants, and marine structures which, if they fail, can cause serious human, environmental and financial losses. The economic and humanitarian aspects of $\mathrm{H}$-induced cracking failures have led to considerable scientific and engineering efforts directed at understanding and preventing such failures. More recently, materials problems

Please enter \offprintinfo\{(Title, Edition)\}\{(Author) \} 
for emerging $\mathrm{H}$ economy have attracted great attentions, such as materials for fuel cells, hydrogen storage and production, etc. All these applications require fundamental understanding of H-metal interactions. In particular, the mechanical properties of the materials are crucial for the success of the relevant applications.

In the past four decades, intense research on $\mathrm{H}$ embrittlement has been carried out and three general mechanisms of $\mathrm{H}$ embrittlement have been put forward, including (1) stressinduced hydride formation and cleavage of the brittle phase [3, 4, 5], (2) $\mathrm{H}$ enhanced local plasticity $[6,7,8,9]$ and (3) $\mathrm{H}$-induced de-cohesion and grain boundary weakening $[10,11,12]$. However, despite the impressive progress, the underlying atomic processes and the relative importance of the three mechanisms remain uncertain and controversial. It is fair to say that a complete mechanistic understanding of $\mathrm{H}$ embrittlement still eludes us [1].

Some of the most fascinating problems in all fields of science involve multiple spatial and/or temporal scales: processes that occur at a certain scale govern the behavior of the system across several (usually larger) scales [13]. In many problems of materials science this notion arises quite naturally: the ultimate microscopic constituents of materials are ions and electrons; interactions among them at the atomic level (of order nanometers and femtoseconds) determine the behavior of the material at the macroscopic scale (of order centimeters and milliseconds and beyond), the latter being the scale of interest for technological applications [14]. The idea of performing simulations of materials across several characteristic length and time scales has therefore obvious appeal as a tool of potentially great impact on technological innovation. The advent of ever more powerful computers which can handle such simulations provides further argument that such an approach can address realistic situations and can be a worthy partner to the traditional approaches of theory and experiment $[15,16,17]$. At each length and time-scale, well-established and efficient computational approaches have been developed over the years to handle the relevant phenomena. To treat electrons explicitly and accurately at the atomic scale, methods based on density functional theory (DFT) [18] and local density approximation [19] can be readily applied to systems containing several hundred atoms. For material properties at the microscopic scale, molecular dynamics or statics simulations are usually performed employing classical interatomic potentials. Although not as accurate as the DFT methods, the classical simulations are able to provide insight into atomic processes involving considerably larger systems, reaching up to $10^{9}$ atoms [20]. Finally, for the macroscopic scale, finite element (FE) methods are routinely used to examine the large-scale properties of materials considered as an elastic continuum [21].

The challenge in modern simulations of materials science and engineering is that real materials usually exhibit phenomena at one scale that require a very accurate and computationally expensive description, and phenomena at another scale for which a coarser description is satisfactory and in fact necessary to avoid prohibitively large computations. Since none of the methods above alone would suffice to describe the entire system, the goal becomes to develop models that combine different methods specialized at different scales, effectively distributing the computational power where it is needed most. It is the hope that a multiscale approach is the answer to such a quest. Sometimes a full-blown brute force calculation could shadow the crucial physics owing to its sheer amount of information or complexity. On the other hand, effective theories and well-constructed multiscale models could capture essential physics without the distraction of less important details [13, 14]. Overall, multiscale modeling is a vibrant enterprise of multi-disciplinary nature. It combines the skills of physicists, materials scientists, chemists, mechanical and chemical engineers, applied mathematicians and computer scientists. The marriage of disciplines and 
the concomitant dissolution of traditional barriers between them represent the true power and embody the great promise of multiscale approaches for enhancing our understanding of, and our ability to control complex physical phenomena [14].

Modeling $\mathrm{H}$ embrittlement is an incredibly challenging task that requires sophisticated multiscale modeling. For example, $\mathrm{H}$ assisted cracking is at the heart of $\mathrm{H}$ embrittlement. To modeling it, one has to involve $a b$ initio-based multiscale approaches. This is because on one hand, a crack propagates by breaking atomic bonds at the crack tip, thus the atomic process of $\mathrm{H}$ attacking metallic bonds has to be captured quantum mechanically. On the other hand, the crack is loaded remotely from exterior surfaces that are far away from the crack tip. Hence the processes that transmit and magnify the applied load to the crack tip have to be accounted for, which can be modeled most effectively by continuum mechanics. Similarly, the interactions between $\mathrm{H}$ and other extended defects such as dislocations, grain boundaries, voids, etc, inevitably require $a b$ initio-based multiscale modeling. Therefore, owing to the inherent multiscale nature of $\mathrm{H}$ embrittlement, $a b$ initio-based multiscale approaches are the most promising theoretical means to address outstanding experimental and theoretical problems in $\mathrm{H}$ embrittlement. In this chapter, we present several $a b$ initio multiscale modeling approaches that we have developed recently and have been used to study crucial problems in $\mathrm{H}$ embrittlement of metals.

In the following, we first provide an introduction to the multiscale methods, including Peierls-Nabarro model for dislocations, quantum mechanical/molecular mechanical (QM/MM) methods and quasi-continuum density functional theory (QCDFT) method. We then apply these methods to study some key phenomena and processes in $\mathrm{H}$ embrittlement of metals, ranging from $\mathrm{H}$-enhanced local plasticity (HELP), $\mathrm{H}$-assisted cracking, crucial role of vacancies to $\mathrm{H}$ diffusion in extended defects. Finally, we propose a tentative mechanism for $\mathrm{H}$ embrittlement and speculate on one future research direction in this area that is interesting to us. The materials presented here primarily reflect our own research interests in multiscale modeling of $\mathrm{H}$ embrittlement, and they are by no means exhaustive. Nevertheless, we hope that they offer a glimpse of the current modeling effort of the field and perhaps can serve as an inspiration for future work in this area.

\subsection{Multiscale Modeling Approaches}

Conceptually, two categories of multiscale modeling approaches can be envisioned - sequential and concurrent approaches [14]. The sequential modeling approaches attempt to piece together a hierarchy of computational methods in which large-scale models use the coarse-grained representations with information obtained from more detailed, smallerscale models. The sequential modeling approaches have proven effective in systems in which the different scales are only weakly coupled. The concurrent approaches on the other hand attempt to link models appropriate at each scale together in a combined theory, where the different scales of the system are considered concurrently and communicate with some type of hand-shaking procedure. These approaches are necessary for systems that are inherently multiscale; that is, systems whose behavior at each scale depends strongly on what happens at the other scales. One important advantage of the concurrent approaches is that they do not require a priori knowledge of the system of interest, neither do they rely on phenomenological models. Thus the concurrent approaches are particularly useful to explore problems about which little is known at the atomistic level and its connection to larger scales, and to discover new phenomena. 


\subsubsection{Peierls-Nabarro Model of Dislocations}

The Peierls-Nabarro (P-N) model was first proposed by Peierls [22] and Nabarro [23] to incorporate the details of a discrete dislocation core into a framework that is essentially a continuum. More specifically, the atomistic scale information of the P-N model is contained in the form of so-called generalized stacking fault energy surface (also referred to as the $\gamma$-surface) and the higher scale information is described by a phenomenological continuum description. In fact, the former can be determined quantum mechanically by $a b$ initio calculations. The P-N model serves as a link between atomistic and continuum approaches, by providing a means to incorporate information obtained from atomistic calculations ( $a b$ initio or empirical) directly into continuum models. The resultant approach can then be applied to problems that neither atomistic nor conventional continuum models could handle separately. The simplicity of the P-N model makes it an attractive alternative to direct atomistic simulations of dislocation properties [24, 25, 26, 27, 28, 29, 30, 31, 32, $33,34,35,36]$. It provides a rapid and inexpensive route to determine dislocation core structure and mobility. Combined with ab initio determined GSF energy surface, the P-N model could give rather reliable quantitative predictions for various dislocation properties.

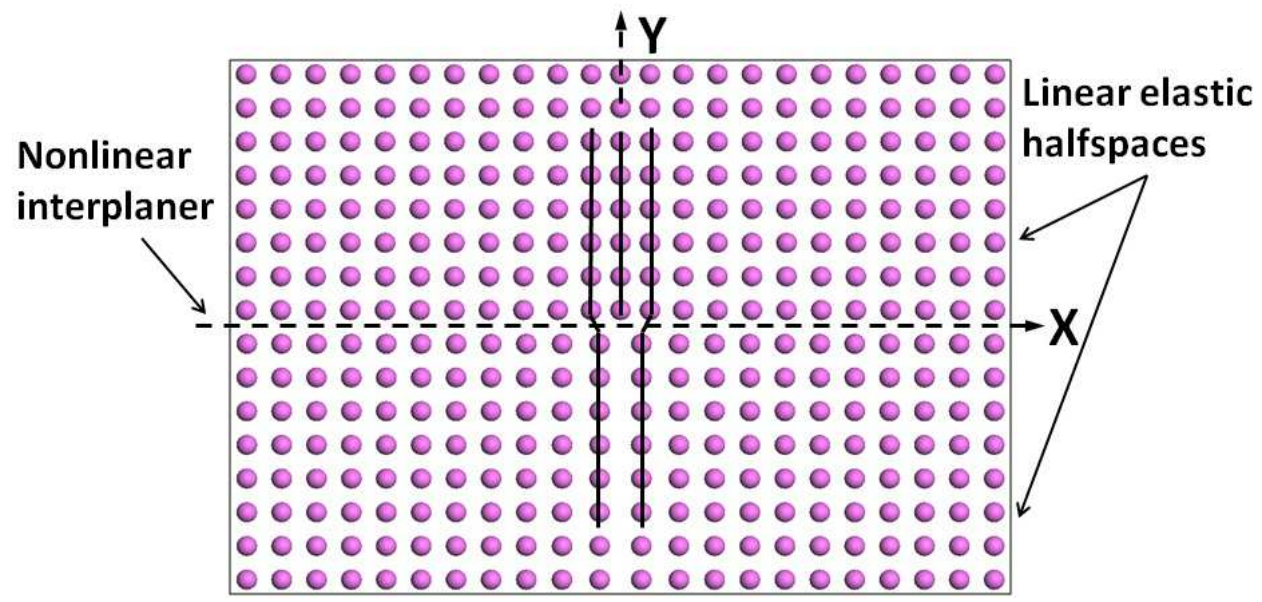

Figure 1.1 A schematic illustration showing an edge dislocation in a lattice. The partition of the dislocated lattice into a linear elastic region and a nonlinear atomistic region allows a multiscale treatment of the problem.

Consider a solid with an edge dislocation in the middle as shown in Fig. 1.1: the solid containing this dislocation is represented by two elastic half-spaces joined by atomic-level forces across their common interface, known as the glide plane (dashed line). The goal of the P-N model is to determine the slip distribution on the glide plane, which minimizes the total energy. The dislocation is characterized by the slip (relative displacement) distribution

$$
\mathbf{f}(x)=\mathbf{u}\left(x, 0^{+}\right)-\mathbf{u}\left(x, 0^{-}\right),
$$

which is a measure of the misfit across the glide plane; $\mathbf{u}\left(x, 0^{+}\right)$and $\mathbf{u}\left(x, 0^{-}\right)$are the displacement of the half-spaces at position $x$ immediately above and below the glide plane. The total energy of the dislocated solid includes two contributions: the nonlinear potential energy resulting from the atomistic interaction across the glide plane, and the elastic energy stored in the two half-spaces associated with the presence of the dislocation. Both energies 
are functionals of the slip distribution $\mathbf{f}(x)$. Specifically, the nonlinear misfit energy can be written as

$$
U_{\text {misfit }}=\int_{-\infty}^{\infty} \gamma[\mathbf{f}(x)] d x
$$

where $\gamma(\mathbf{f})$ is the generalized stacking fault energy surface introduced by Vitek [37]. The nonlinear interplanar $\gamma$-surface can be determined from ab initio calculations. The elastic energy of the dislocation can be calculated reasonably from elasticity theory: the dislocation may be thought of as a continuous distribution of infinitesimal dislocations whose Burgers vectors integrate to that of the original dislocation [38]. Therefore, the elastic energy of the original dislocation is just the sum of the elastic energy caused by all the infinitesimal dislocations (from the superposition principle of linear elasticity theory), which can be written as

$$
U_{\text {elastic }}=\frac{\mu}{2 \pi(1-\nu)} \int d x \int d x^{\prime} \ln \frac{L}{\left|x-x^{\prime}\right|} \frac{d \mathbf{f}(x)}{d x} \frac{d \mathbf{f}\left(x^{\prime}\right)}{d x^{\prime}}
$$

where $\mu$ and $\nu$ are the shear modulus and Poisson's ratio, respectively. The variable $L$ is an inconsequential constant introduced as a large-distance cutoff for the computation of the logarithmic interaction energy [39]. The gradient of $\mathbf{f}(x)$ is called dislocation (misfit) density, denoted by $\rho(x)$. The successful application of the $\mathrm{P}-\mathrm{N}$ models depends on the reliability of both $\gamma$-surface and the underlying elasticity theory, which is the basis for the formulation of the phenomenological theory.

The total energy of the dislocation is a function of misfit distribution $\mathbf{f}(x)$ or, equivalently, $\rho(x)$, and it is invariant with respect to arbitrary translation of $\rho(x)$ and $\mathbf{f}(x)$. To regain the lattice discreteness, the integration of the $\gamma$-energy in Eq. (1.2) was discretized and replaced by a lattice sum in the original $\mathrm{P}-\mathrm{N}$ formulation

$$
U_{\text {misfit }}=\sum_{i=-\infty}^{\infty} \gamma\left[\mathbf{f}\left(x_{i}\right)\right] \Delta x,
$$

with $x_{i}$ the reference position and $\Delta x$ the average spacing of the atomic rows in the lattice. This procedure, however, is inconsistent with evaluation of elastic energy in Eq. (1.3) as a continuous integral. Therefore, the total energy is not variational. Furthermore, in the original P-N model, the shape of the solution $\mathbf{f}(x)$ is assumed to be invariant during dislocation translation, a problem that is also associated with the non-variational formulation of the total energy.

To resolve these problems, a so-called semidiscrete variational P-N (SVPN) model was developed [31] that allows the study of narrow dislocations, a situation that the standard $\mathrm{P}-\mathrm{N}$ model can not handle. Within this approach, the equilibrium structure of a dislocation is obtained by minimizing the dislocation energy functional

$$
U_{\text {disl }}=U_{\text {elastic }}+U_{\text {misfit }}+U_{\text {stress }}+K b^{2} \ln L,
$$

where

$$
\begin{gathered}
U_{\text {elastic }}=\sum_{i, j} \frac{1}{2} \chi_{i j}\left[K_{e}\left(\rho_{i}^{(1)} \rho_{j}^{(1)}+\rho_{i}^{(2)} \rho_{j}^{(2)}\right)+K_{s} \rho_{i}^{(3)} \rho_{j}^{(3)}\right], \\
U_{\text {misfit }}=\sum_{i} \gamma_{3}\left[\mathbf{f}\left(x_{i}\right)\right] \Delta x,
\end{gathered}
$$




$$
U_{\text {stress }}=-\sum_{i, l} \frac{x_{i}^{2}-x_{i-1}^{2}}{2} \rho_{i}^{(l)} \tau_{i}^{(l)},
$$

with respect to the dislocation misfit density. Here, $\rho_{i}^{(1)}, \rho_{i}^{(2)}$, and $\rho_{i}^{(3)}$ are the edge, vertical, and screw components of the general interplanar misfit density at the $i$ th nodal point, respectively, and $\gamma_{3}(\mathbf{f})$ is the corresponding three-dimensional $\gamma$-surface. The components of the applied stress are $\tau^{(1)}=\sigma_{21}, \tau^{(2)}=\sigma_{22}$, and $\tau^{(3)}=\sigma_{23}$, respectively. The variables $K, K_{e}$, and $K_{s}$ are the pre-logarithmic elastic energy factors, related to the shear modulus, Poisson's ratio, and the dislocation character [39]. The dislocation density at the $i$ th nodal point is $\left.\rho_{i}=(f) i-f_{i-1}\right) /\left(x_{i}-x_{i-1}\right)$, and $\chi_{i j}$ is the elastic energy kernel [31].

The first term in the energy functional $U_{\text {elastic }}$ is now discretized to be consistent with the discretized misfit energy, which makes the total energy functional variational. Another modification in this approach is that the nonlinear misfit potential in the energy functional $U_{\text {misfit }}$ is a function of all three components of the nodal misfit $\mathbf{f}\left(x_{i}\right)$. Namely, in addition to the misfit along the Burgers vector, lateral and even vertical misfits across the glide plane are also included. This allows for the treatment of straight dislocations of arbitrary orientation in arbitrary glide planes [33,32]. Furthermore, because the misfit vector $\mathbf{f}\left(x_{i}\right)$ is allowed to change during the process of dislocation translation, the energy barrier (referred to as the Peierls barrier) can be significantly lowered compared to the corresponding value taken from a rigid translation. The response of a dislocation to an applied stress is achieved by minimization of the energy functional with respect to $\rho_{i}$ at the given value of the applied stress $\tau_{i}^{(l)}$. An instability is reached when an optimal solution for $\rho_{i}$ no longer exists, which is manifested numerically by the failure of the minimization procedure to converge. The Peierls stress is defined as the critical value of the applied stress that gives rise to this instability. The SVPN model has been applied to study various interesting material problems related to dislocation phenomena $[33,32,34,35,36]$.

\subsubsection{Quantum mechanics/molecular mechanics method}

In atomistic modeling of materials, quantum mechanics $(\mathrm{QM})$ is necessary for a proper treatment of phenomena such as bond-breaking, charge transfer, electronic and optical excitations and magnetism, etc; however, owing to its computational demand, the application of QM has to be restricted to relatively small systems consisting of up to a few hundreds of atoms. On the other hand, atomistic simulations based on empirical interatomic potentials are often capable of describing small-amplitude vibrations and torsions, elastic deformation, electrostatic interactions, etc., in many materials. Termed as molecular mechanical (MM) methods, these empirical atomistic approaches can treat millions of atoms or more. Therefore algorithms that combine quantum mechanics and molecular mechanics (QM/MM) poise to offer a promising solution to the computational challenge in atomistic simulations of materials [40, 41, 42].

For many molecular systems that are of interest in chemistry and biochemistry, one can partition the QM/MM system by cutting chemical bonds linking the QM and MM parts and then saturate the dangling bonds at the boundary of QM region by so-called link atoms $[41,43,44]$. This procedure and the similar ones can be justified because of the presence of well-defined and localized chemical bonds in such molecular systems. Unfortunately, for metallic materials, the procedure is no longer valid owing to the delocalized nature of metallic bonding; it becomes impractical to cut and saturate bonds. In fact, the very concept of chemical bonds becomes less appropriate than the band picture for metals. Therefore, more sophisticated ideas have to be developed to deal with metallic cohesion represented 
by the delocalized electron states across the QM/MM boundary. As a result, far fewer QM/MM-like simulations have been attempted in metallic systems [42, 45, 46, 47, 48, 49]. Here, we present some recent development of such ideas for modeling metallic materials [50, 51, 52, 53, 42].

In a QM/MM simulation, the computational domain is partitioned into two regions: region I is the primary region where QM simulations are performed and region II is the surrounding region where classical atomistic simulations are carried out. Although different levels of QM simulations could be employed in region I, here we focus on the Kohn-Sham density functional theory (KS-DFT) [19]. Similarly, although many empirical potentials could be used in region II, we choose the embedded-atom method (EAM) [54] as an example for MM calculations. The total energy of a system including the energy of the region I, the energy for the region II, and the interaction energy between them can be expressed as

$$
E_{\text {tot }}[\mathrm{I}+\mathrm{II}]=E_{\mathrm{DFT}}[\mathrm{I}]+E^{\mathrm{int}}[\mathrm{I}, \mathrm{II}]+E_{\mathrm{EAM}}[\mathrm{II}] .
$$

The interaction between regions I and II is at the heart of any QM/MM method. Depending on the formulation of the interaction energy, the QM/MM methods can be divided into two categories: mechanical coupling and quantum coupling. In the quantum coupling, the interaction energy $E^{\text {int }}[\mathrm{I}, \mathrm{II}]$ is formulated by DFT; the quantum mechanical calculation for region I is carried out in the presence of region II; and a single-particle embedding potential that represents the quantum mechanical effects of region II enters the QM Hamiltonian of region I. On the other hand, in the mechanical coupling, one performs quantum mechanical simulation of region I in the absence of region II, and treats the interaction energy $E^{\text {int }}[\mathrm{I}, \mathrm{II}]$ at the MM level.

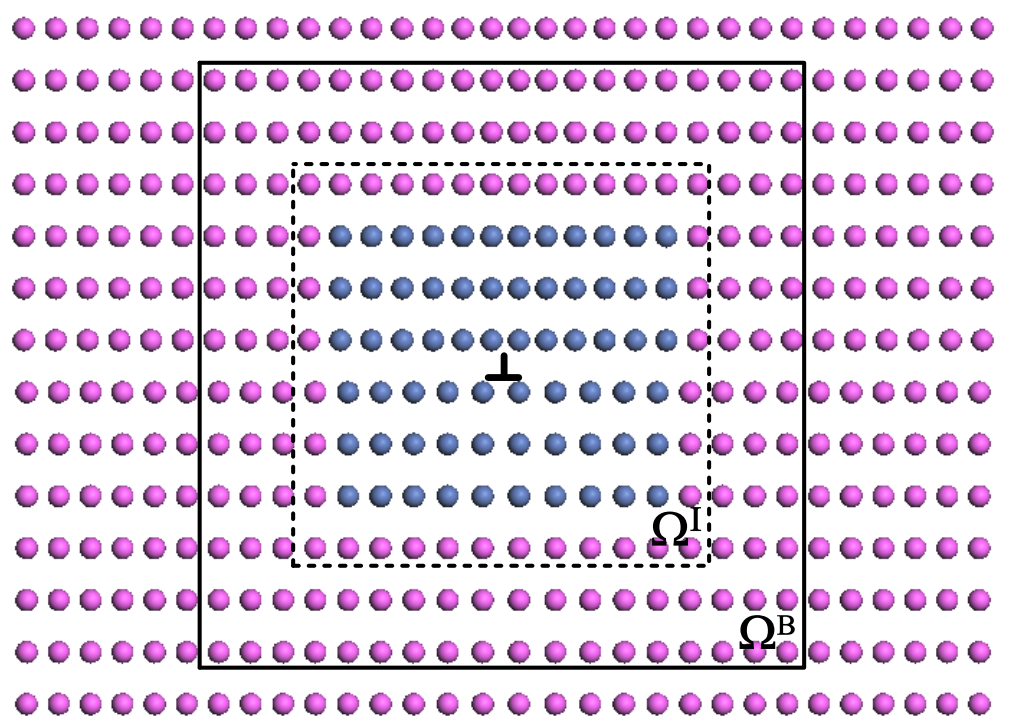

Figure 1.2 Schematic domain partition in the QM/MM method with an edge dislocation as an example. Blue and magenta spheres represent the atoms belonging to region I and region II, respectively. The dashed box represents $\Omega^{\mathrm{I}}$, and the solid box represents the periodic box $\Omega^{\mathrm{b}}$. The charge density of region I $\rho^{\mathrm{I}}$ is confined within $\Omega^{\mathrm{I}}$, and the periodic boundary conditions are imposed over $\Omega^{\mathrm{b}}$. From reference [53]. 
1.2.2.1 Quantum Coupling The self-consistent determination of the interaction energy at a QM level is the hallmark of the quantum coupling approach. The spatial partition of the entire system is shown in Fig. 1.2. In specific, the total energy can be expressed as

$$
E_{\text {tot }}\left[\rho^{\text {tot }} ; \mathbf{R}^{\text {tot }}\right]=\min _{\rho^{\mathrm{I}}}\left\{E_{\mathrm{KS}}\left[\rho^{\mathrm{I}} ; \mathbf{R}^{\mathrm{I}}\right]+E_{\mathrm{OF}}^{\mathrm{int}}\left[\rho^{\mathrm{I}}, \rho^{\mathrm{II}} ; \mathbf{R}^{\mathrm{I}}, \mathbf{R}^{\mathrm{II}}\right]\right\}+E_{\mathrm{MM}}\left[\mathbf{R}^{\mathrm{II}}\right],
$$

where $\mathbf{R}^{\text {tot }} \equiv \mathbf{R}^{\mathrm{I}} \cup \mathbf{R}^{\mathrm{II}}, \mathbf{R}^{\mathrm{I}}$ and $\mathbf{R}^{\mathrm{II}}$ denote atomic coordinates in region I and II respectively. The charge density of region $\mathrm{I}, \rho^{\mathrm{I}}$, which is the degree of the freedom of the problem, is determined self-consistently by minimizing the total energy functional Eq. (1.10). We associate each MM atom in region II with an atomic-centered electron density $\left(\rho^{\text {at }}\right)$ and a pseudopotential; both of them are constructed a priori. The charge density of region II, $\rho^{\mathrm{II}}$, is defined as a superposition of atomic-centered charge densities $\rho^{\text {at }}$ via $\rho^{\mathrm{II}}(\mathbf{r})=\sum_{i \in \mathrm{II}} \rho^{\text {at }}\left(\mathbf{r}-\mathbf{R}_{i}\right)$, which only changes upon the relaxation of region II ions. The total charge density $\rho^{\text {tot }}$ is given by $\rho^{\text {tot }}=\rho^{\mathrm{I}}+\rho^{\mathrm{II}}$. The interaction energy between region I and II, $E_{\mathrm{OF}}^{\mathrm{int}}$, formulated by Orbital-Free Density Functional Theory (OF-DFT) $[56,57,58]$ is defined as follows

$$
E_{\mathrm{OF}}^{\mathrm{int}}\left[\rho^{\mathrm{I}}, \rho^{\mathrm{II}} ; \mathbf{R}^{\mathrm{I}}, \mathbf{R}^{\mathrm{II}}\right]=E_{\mathrm{OF}}\left[\rho^{\mathrm{tot}} ; \mathbf{R}^{\mathrm{tot}}\right]-E_{\mathrm{OF}}\left[\rho^{\mathrm{I}} ; \mathbf{R}^{\mathrm{I}}\right]-E_{\mathrm{OF}}\left[\rho^{\mathrm{II}} ; \mathbf{R}^{\mathrm{II}}\right] .
$$

The unique feature of OF-DFT is that it allows a QM calculation of energetics by knowing only the charge density. The accuracy of OF-DFT is in between KS-DFT and EAM, which is consistent to its usage in the QM/MM method. Finally, the EAM method was developed specifically to treat metallic systems. $\mu_{\mathrm{emb}}\left[\rho^{\mathrm{I}}, \rho^{\mathrm{II}}\right]$ termed as embedding potential, is of crucial importance to the QM/MM method, and is defined as a functional derivative of the interaction energy $E_{\mathrm{OF}}^{\text {int }}$ with respect to $\rho^{\mathrm{I}}$,

$$
\mu_{\mathrm{emb}}(\mathbf{r}) \equiv \frac{\delta E_{\mathrm{OF}}^{\mathrm{int}}\left[\rho^{\mathrm{I}}, \rho^{\mathrm{II}} ; \mathbf{R}^{\mathrm{I}}, \mathbf{R}^{\mathrm{II}}\right]}{\delta \rho^{\mathrm{I}}} .
$$

$\mu_{\mathrm{emb}}(\mathbf{r})$ represents the effective single-particle potential that the region I electrons feel due to the presence of the region II atoms (both electrons and ions). We have recently developed a quantum coupling approach in which the interaction energy is calculated by KS-DFT, thus overcomes the limitations of OF-DFT. Based on the constrained DFT, the method is more general and is particularly useful for magnetic materials [55].

1.2.2.2 Mechanical coupling In the mechanical coupling, the interaction energy $E^{\text {int }}$ is evaluated with EAM calculations,

$$
E^{\text {int }}[\mathrm{I}, \mathrm{II}]=E_{\mathrm{EAM}}[\mathrm{I}+\mathrm{II}]-E_{\mathrm{EAM}}[\mathrm{I}]-E_{\mathrm{EAM}}[\mathrm{II}],
$$

and thus Eq. (1.9) becomes

$$
E_{\mathrm{tot}}[\mathrm{I}+\mathrm{II}]=E_{\mathrm{DFT}}[\mathrm{I}]+E_{\mathrm{EAM}}[\mathrm{I}+\mathrm{II}]-E_{\mathrm{EAM}}[\mathrm{I}] .
$$

The advantage of the mechanical coupling is simplicity [59]. It demands nothing beyond what is required for a DFT cluster calculation and two classical MM calculations (one for the entire system and the other for a cluster of region I). On the other hand, the quantum coupling allows a more accurate description, in particular, for the electronic structure of region I. For example, the fictitious surface states due to the cluster calculation of region I in the mechanical coupling can be eliminated in the quantum coupling [52]. Of course, the quantum coupling is more complicated than the mechanical coupling in general. 


\subsubsection{Quasi-continuum density functional theory}

A widely used and successful concurrent multiscale method is so-called quasi-continuum (QC) method originally proposed by Tadmor et al. [60]. The QC method combines atomistic models with continuum theories, and thus offers an advantage over conventional atomistic simulations in terms of computational efficiency. The idea underlying the QC method is that atomistic processes of interest often occur in very small spatial domains (e.g., crack tip) while the vast majority of atoms in the material behave according to well-established continuum theories. To exploit this fact, the QC method retains atomic resolution only where necessary and coarsens to a continuum finite-element description elsewhere. This is achieved by replacing the full set of $N$ atoms with a small subset of $N_{\mathrm{r}}$ "representative atoms" or repatoms $\left(N_{\mathrm{r}} \ll N\right)$ that approximate the total energy through appropriate weighting. The energies of individual repatoms are computed in two different ways depending on the deformation in their immediate vicinity. Atoms experiencing large deformation gradients on an atomic-scale are computed in the same way as in a standard fully-atomistic method. In QC these atoms are called nonlocal atoms. In contrast, the energies of atoms experiencing a smooth deformation field on the atomic scale are computed based on the deformation gradient in their vicinity as befitting a continuum model. These atoms are called local atoms. The total energy $E_{\text {tot }}$ (which for a classical system can be written as $E_{\mathrm{tot}}=\sum_{i=1}^{N} E_{i}$, with $E_{i}$ the energy of atom $i$ ) is approximated as

$$
E_{\mathrm{tot}}^{\mathrm{QC}}=\sum_{i=1}^{N_{\mathrm{n} 1}} E_{i}(\{\mathbf{q}\})+\sum_{j=1}^{N_{\mathrm{loc}}} n_{j} E_{j}^{\mathrm{loc}}(\{\mathbf{F}\}) .
$$

The total energy has been divided into two parts: an atomistic region of $N_{\mathrm{nl}}$ nonlocal atoms and a continuum region of $N_{\text {loc }}$ local atoms $\left(N_{\mathrm{nl}}+N_{\text {loc }}=N_{\mathrm{r}}\right)$.

The original formulation of QC was limited to classical potentials for describing interactions between atoms. However, since many materials properties depend crucially on the behavior of electrons, such as bond breaking/forming at crack tips or defect cores, chemical reactions with impurities, surface reactions and reconstructions, and magnetism, etc, it is desirable to incorporate appropriate quantum mechanical descriptions into the QC formalism. QCDFT is one strategy to fill this role. In specific, QCDFT combines the coarse graining idea of QC and the coupling strategy of QM/MM approaches mentioned above $[61,62,63]$. Therefore QCDFT can capture the electronic structure at the defect cores (e.g. crack tip) within the accuracy of DFT and at the same time reach the length-scale that is relevant to experiments.

The original QC formulation assumes that the total energy can be written as a sum over individual atom energies. This condition is not satisfied by quantum mechanical models. To address this limitation, in the present QCDFT approach the nonlocal region is treated by either the mechanical or the quantum coupling QM/MM approaches [61, 62]. Here, for simplicity, we only discuss the mechanical coupling QCDFT in which the nonlocal QC formulation is based on the coupling between KS-DFT and EAM with the interaction energy determined by EAM. The local region, on the other hand, is also dealt with by EAM. This makes the passage from the atomistic to continuum seamless since the same underlying material description is used in both. This description enables the model to adapt automatically to changing circumstances (e.g. the nucleation of new defects or the migration of existing defects). The adaptability is one of the main strengths of QCDFT, which is missing in many other multiscale methods. 


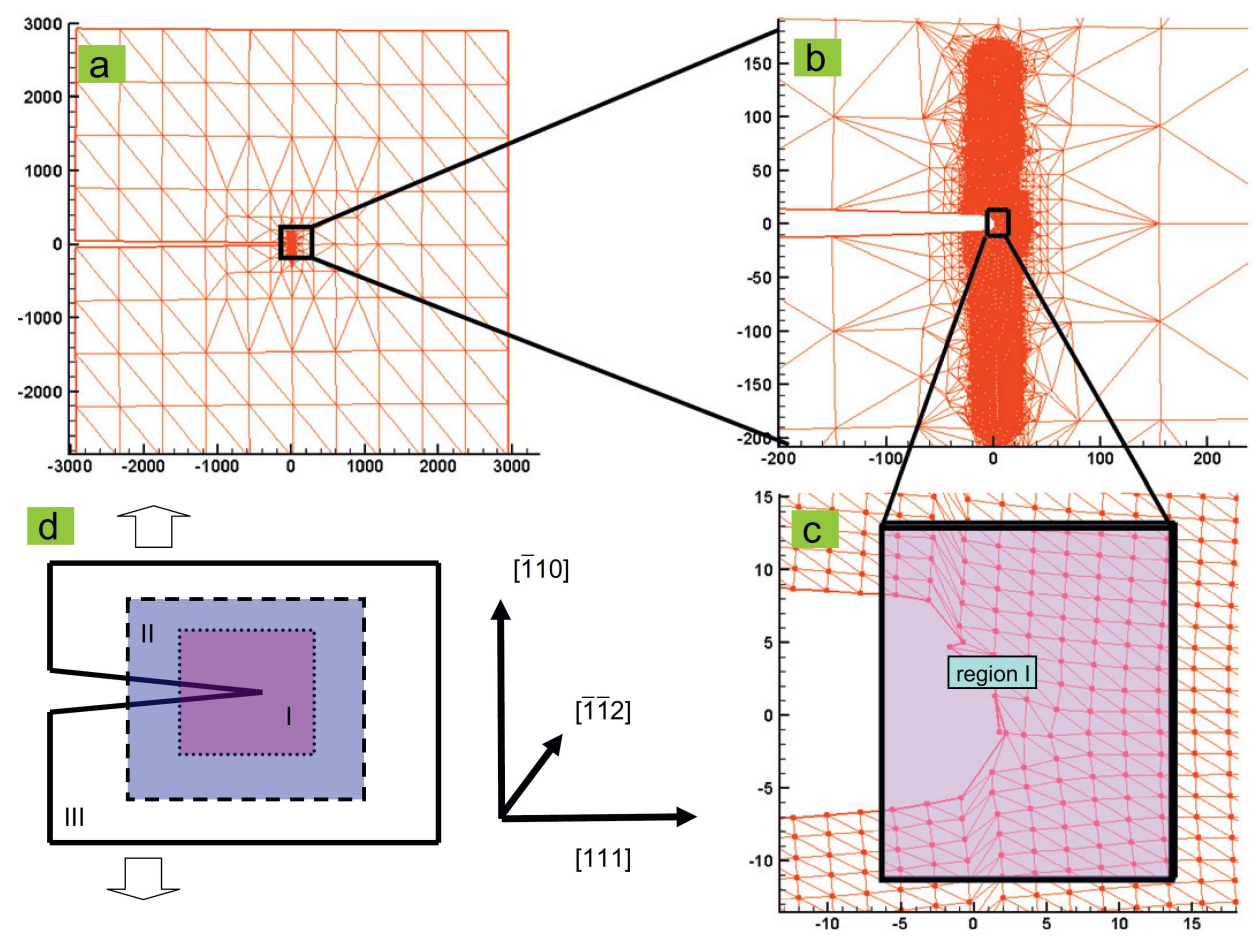

Figure 1.3 QCDFT model for a crack showing the three domains in the model. (a) The overview of the entire crack system with finite-element mesh; (b) a blown-up view of (a) showing the nonlocal region; (c) region I box and atomic structure at the crack tip; (d) schematic partition of the system into regions I, II and III. From reference [63].

More specifically, in the QCDFT approach the material of interest is partitioned into three distinct domains as shown in Fig. 1.3: (1) a nonlocal quantum mechanical DFT region (region I); (2) a nonlocal classical region where classical EAM potentials are used (region II); and (3) a local region (region III) that employs the same EAM potentials as region II. The coupling between region II and III is achieved via the QC formulation, while the coupling between region I and II is accomplished by the QM/MM scheme [59]. The total energy of the QCDFT system is thus given by [61]

$$
\begin{aligned}
E_{\mathrm{tot}}^{\mathrm{QCDFT}} & =E^{\mathrm{nl}}[\mathrm{I}+\mathrm{II}]+\sum_{j=1}^{N_{\mathrm{loc}}} n_{j} E_{j}^{\mathrm{loc}}(\{\mathbf{F}\}) \\
& =E_{\mathrm{DFT}}[\mathrm{I}]-E_{\mathrm{EAM}}[\mathrm{I}]+E_{\mathrm{EAM}}[\mathrm{I}+\mathrm{II}]+\sum_{j=1}^{N_{\mathrm{loc}}} n_{j} E_{j}^{\mathrm{loc}}(\{\mathbf{F}\}),
\end{aligned}
$$

where $E^{\mathrm{nl}}[\mathrm{I}+\mathrm{II}]$ is the total energy of the nonlocal region (I and II combined with the assumption that region I is embedded within region II), $E_{\mathrm{DFT}}[\mathrm{I}]$ is the energy of region I in the absence of region II computed with DFT, $E_{\mathrm{EAM}}[\mathrm{II}]$ is the energy of region II in the absence of region I computed with EAM, and $E_{\mathrm{EAM}}[\mathrm{I}+\mathrm{II}]$ is the energy of the nonlocal region computed with EAM. 
Other combinations of quantum mechanical and classical atomistic methods may also be implemented in QCDFT. The advantage of the present implementation is its simplicity; it has nothing more than a DFT calculation and an EAM-QC calculation. Another advantage of the QCDFT method is that, if region I contains multiple atomic species while region II contains only one atom type, there is no need to develop reliable EAM potentials to describe each species and their interactions. This is because if the various species of atoms are well within region I, the energy contributions of these atoms are canceled out in the total energy calculation. This advantage renders the method useful in dealing with impurities, which is an exceedingly difficult task for conventional empirical simulations.

The equilibrium structure of the system is obtained by minimizing the total energy in Eq. (1.16) with respect to all degrees of freedom. Because the time required to evaluate $E_{\mathrm{DFT}}[\mathrm{I}]$ is considerably more than that required for computation of the other EAM terms in $E_{\text {tot }}^{Q C D F T}$, an alternate relaxation scheme turns out to be useful. The total system can be relaxed by using conjugate gradient approach on the DFT atoms alone, while fully relaxing the EAM atoms in region II and the displacement field in region III at each step. An auxiliary energy function can be defined as

$$
E^{\prime}\left[\left\{\mathbf{q}^{\mathrm{I}}\right\}\right] \equiv \min _{\left\{\mathbf{q}^{\mathrm{II}}\right\},\left\{\mathbf{q}^{\mathrm{III}}\right\}} E_{\mathrm{tot}}^{\mathrm{QCDFT}}[\{\mathbf{q}\}]
$$

which allows for the following relaxation scheme: (i) minimize $E_{\text {tot }}^{\mathrm{QCDFT}}$ with respect to the atoms in regions II $\left(\left\{\mathbf{q}^{\mathrm{II}}\right\}\right)$ and the atoms in region III $\left(\left\{\mathbf{q}^{\mathrm{III}}\right\}\right)$, while holding the atoms in region I fixed; (ii) calculate $E_{\text {tot }}^{\mathrm{QCDFT}}[\{\mathbf{q}\}]$, and the forces on the region I atoms; (iii) perform one step of conjugate gradient minimization of $E^{\prime}$; (iv) repeat until the system is relaxed. In this manner, the number of DFT calculations performed is greatly reduced, albeit at the expense of more EAM and local QC calculations. It has been shown shown that the total number of DFT energy calculations for the relaxation of an entire system is about the same as that required for DFT relaxation of region I alone.

\subsection{Multiscale Modeling of Hydrogen Embrittlement}

In the past decade, we have used multiscale modeling to study some key problems in $\mathrm{H}$ embrittlement of metals by focusing on $\mathrm{H}$-defect interactions, which are summarized below.

\subsubsection{Hydrogen Enhanced Local Plasticity}

In recent years, a unified understanding of $\mathrm{H}$ embrittlement is emerging based on the key concept of hydrogen enhanced local plasticity (HELP) [1]. There is a large body of experimental evidence suggesting that $\mathrm{H}$ strongly affects plastic deformation in metals in a manner leading to enhanced fracture. One of the most convincing experimental evidence of HELP is the controlled in situ TEM observation that is performed in real time and at high spatial resolution [7]. Observations were made on specimens that were under stress, but the deformation processes had ceased to operate in vacuum. On adding $\mathrm{H}_{2}$ gas to the environmental cell, dislocations began to move. Subsequently removal of the $\mathrm{H}_{2}$ gas caused a cessation of the dislocation motion, and the cycle could be repeated many times. This $\mathrm{H}$ enhanced dislocation mobility has been observed for screw, edge, and mixed dislocations as well as for isolated dislocations and dislocation tangles. Observations have been made in a wide range of materials with different crystal structures, including $\mathrm{Fe}, \mathrm{Ni}$, steels, $\mathrm{Al}$ 
and $\mathrm{Al}$ alloys, $\mathrm{Ti}$ and Ti-alloys, and $\mathrm{Ni}_{3} \mathrm{Al}[5,64,8,9,65,66,67,68,69,70]$. Another important result, which is believed to be responsible for $\mathrm{H}$ embrittlement is $\mathrm{H}$-induced slip planarity. It has been revealed by microscopic observations that solute $\mathrm{H}$ atoms can inhibit dislocation cross-slip in $\mathrm{Al}$, austenitic stainless steels, $\mathrm{Ni}, \mathrm{NiCo}$, and Ni-based superalloy, leading to slip planarity $[71,72,73,74,75]$. This slip planarity forces dislocations from a given source to remain localized in narrow slip bands because the relaxation of dislocation pile-ups via cross-slip is suppressed. As a consequence, a micro-crack may be initiated in front of a pile-up when the stress intensity factor reaches its critical value over there. In contrast to the vast body of experimental evidence, theoretical studies of the HELP mechanism are scarce. The SVPN and QM/MM calculations have been performed to gain an understanding of the physics behind the HELP mechanism [34, 53, 76, 77].

In the SVPN approach, ab initio calculations are carried out for for the $\gamma$-surface of $\mathrm{Al}$ with $\mathrm{H}$ impurities placed at the interstitial sites [34]. The $\gamma$-surfaces for both pure $\mathrm{Al}$ and the $\mathrm{Al}+\mathrm{H}$ systems are shown in Fig. 1.4. Comparing the two $\gamma$-surfaces, one finds an overall reduction (up to 50\%) in $\gamma$-energy in the presence of $\mathrm{H}$, which is attributed to the change of atomic bonding across the glide plane, from covalent-like to ionic-like [78]. This reduction of $\gamma$-energy could change dislocation properties significantly. For example, one expects that dislocations would be emitted more easily from a crack tip when $\mathrm{H}$ is present based on the lowering of the unstable stacking fault energy along the [12 1$]$ direction [79]. In fact, this prediction is in accordance with atomistic simulations for $\mathrm{Ni}$, where dislocations are found to be emitted more rapidly from a crack tip in the presence of $\mathrm{H}[80]$.
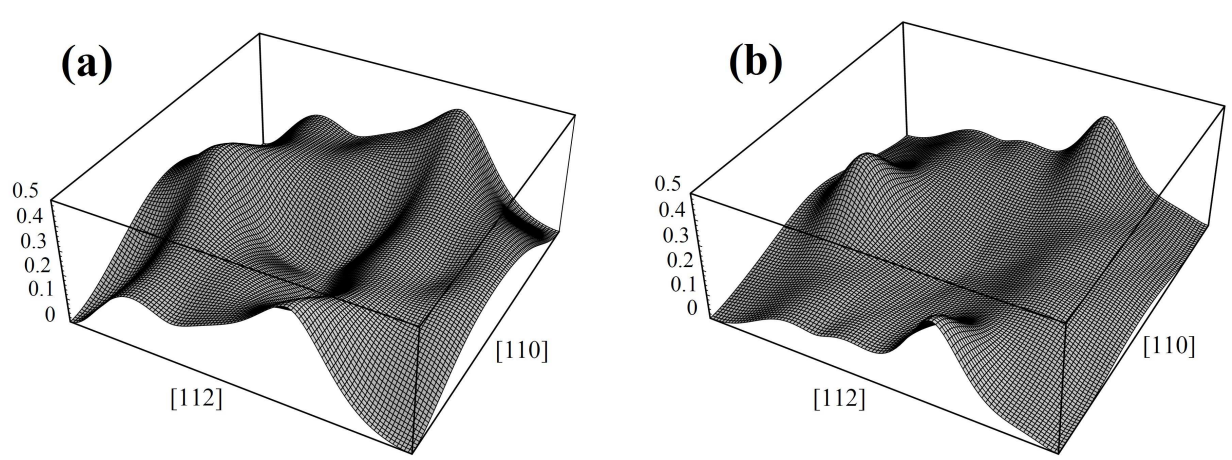

Figure 1.4 The $\gamma$-surface $\left(\mathrm{J} / \mathrm{m}^{2}\right)$ for displacements along a (111) plane for (a) pure $\mathrm{Al}$ and (b) $\mathrm{Al}+$ $\mathrm{H}$ systems. The corners of the plane and its center correspond to identical equilibrium configurations (i.e., the ideal lattice). The two surfaces are displayed in exactly the same perspective and on the same energy scale to facilitate comparison of important features. From reference [34].

The core properties of four different dislocations, screw $\left(0^{\circ}\right), 30^{\circ}, 60^{\circ}$, and edge $\left(90^{\circ}\right)$ have been studied using the SVPN model. The Peierls stress for these dislocations is shown in Table 1.1. It was found that the Peierls stress, which is the minimum stress to move a stationary dislocation, is reduced by more than an order of magnitude in the presence of $\mathrm{H}$. Moreover this H-enhanced mobility is observed for screw, edge, and mixed dislocations, which is compatible with the experimental results. This result invalidates the perception that the only plausible explanation of HELP must be based on elastic interactions among dislocations and that dislocation-lattice interaction is not important [1]. On the contrary, it was found that the dislocation-lattice interaction is very important and that the dislocation 
Table 1.1 Peierls stress $\left(\sigma_{p}, \mathrm{MPa}\right)$, core energies $\left(E_{\text {core }}, \mathrm{ev} / \AA \mathrm{A}\right)$ and binding energy $\left(E_{\mathrm{b}}, \mathrm{eV} /\right.$ atom $)$ for the four dislocations in the pure $\mathrm{Al}$ and the $\mathrm{Al}+\mathrm{H}$ systems. From reference [34].

\begin{tabular}{cccccc}
\hline & & Screw & $30^{\circ}$ & $60^{\circ}$ & Edge \\
\hline$\sigma_{p}$ & $\mathrm{Al}$ & 254 & 51 & 97 & 3 \\
& $\mathrm{Al}+\mathrm{H}$ & 1.7 & 1.2 & 1.4 & 0.3 \\
$E_{\text {core }}$ & $\mathrm{Al}$ & -0.08 & -0.11 & -0.17 & -0.20 \\
& $\mathrm{Al}+\mathrm{H}$ & -0.14 & -0.18 & -0.27 & -0.32 \\
$E_{\mathrm{b}}$ & & -0.06 & -0.07 & -0.10 & -0.12 \\
\hline
\end{tabular}

core structure is responsible for the observed H-enhanced dislocation mobility. However, it does not exclude the possibility that dislocation-dislocation interaction does play a role in HELP [81]. The results of binding energy $E_{\mathrm{b}}$ in Table 1.1 show that there is strong binding between $\mathrm{H}$ and the dislocation cores; that is, $\mathrm{H}$ is attracted (trapped) to dislocation cores, which lowers the core energies. More importantly, the binding energy was found to be a function of dislocation character, with the edge dislocation having the greatest and the screw dislocation having the lowest binding energies. For a mixed dislocation, the binding energy increases with the amount of edge component of the Burgers vector. These results indicate that in the presence of $\mathrm{H}$, it costs more energy for an edge dislocation to transform into a screw dislocation to cross-slip, as the edge dislocation has almost twice the binding energy of the screw dislocation. In the same vein, it costs more energy for a mixed dislocation to transfer its edge component to a screw component for cross-slip. Therefore, the cross-slip process is suppressed because of the presence of $\mathrm{H}$, and the slip is confined to the primary glide plane, exhibiting the experimentally observed slip planarity.

The interaction between the $\mathrm{H}$ impurities with dislocations in $\alpha$-Fe has been examined by using the QM/MM approach [76]. In the QM/MM simulations, the entire dislocation is partitioned into two regions as shown in Fig. 1.2: the QM region contains the dislocation core and the impurities; the MM region on the other hand contains the rest of system including the long-range elastic field of the dislocation. The impurity-dislocation interaction is investigated by calculating the impurity-dislocation solution energy and the impurity binding energy to the dislocation cores. The impurity solution energy is calculated as follows

$$
E_{\mathrm{X}, \mathrm{H}}^{\mathrm{s}}=E_{\mathrm{X}+\mathrm{H}}-E_{\mathrm{X}}-E_{\mathrm{H}} .
$$

Here $\mathrm{X}$ could stand for bulk, edge, screw dislocations respectively. The three energy contributions are the energy of $\mathrm{X}$ in the presence of the $\mathrm{H}$ impurity, the energy of $\mathrm{X}$ in the absence of the $\mathrm{H}$ impurity, and the energy of the $\mathrm{H}$ impurity by itself. The more negative the solution energy, the stronger the binding between $\mathrm{X}$ and $\mathrm{H}$ (or the site is more preferred by the impurity). It was found that in $\alpha$-Fe, $\mathrm{H}$ prefers the tetrahedral (T-) site over the octahedral (O-) site at the dislocation core, as well as in the bulk. We note that the observed site preference of $\mathrm{H}$ is materials specific and thus one could be cautious to generalize the conclusion to other materials. The solution energies of the most stable sites in the bulk, edge, and screw dislocations are $-2.33 \mathrm{eV},-2.80 \mathrm{eV}$, and $-2.60 \mathrm{eV}$, respectively. The impurity binding (or segregation) energy to the dislocation core can be defined as the solution energy difference between the bulk and the dislocations. The lower the binding/segregation energy, the more stable the impurity at the dislocation core; the negative value of the binding/segregation energy indicates that the impurity prefers to segregate to the dislocation core as opposed to staying in the bulk. The lowest binding energies of $\mathrm{H}$ 
to the screw and the edge dislocation are $-0.27 \mathrm{eV}$ and $-0.47 \mathrm{eV}$, respectively. Therefore, the $\mathrm{H}$ impurity is energetically most stable in the edge dislocation, less stable in the screw dislocation and least stable in the bulk, similar to the preceding results of Al. We have also performed QM/MM calculations for $\mathrm{Al}$ dislocations and find a binding energy between $\mathrm{H}$ and the edge dislocation of $-0.12 \mathrm{eV}$ [53], which is identical to the previous P-N model result. However, $\mathrm{H}$ has a weaker binding to dislocations in $\mathrm{Mg}$ where the binding energies are $-0.03 \mathrm{eV}$ and $-0.05 \mathrm{eV}$ for the screw and edge dislocations, respectively [77].

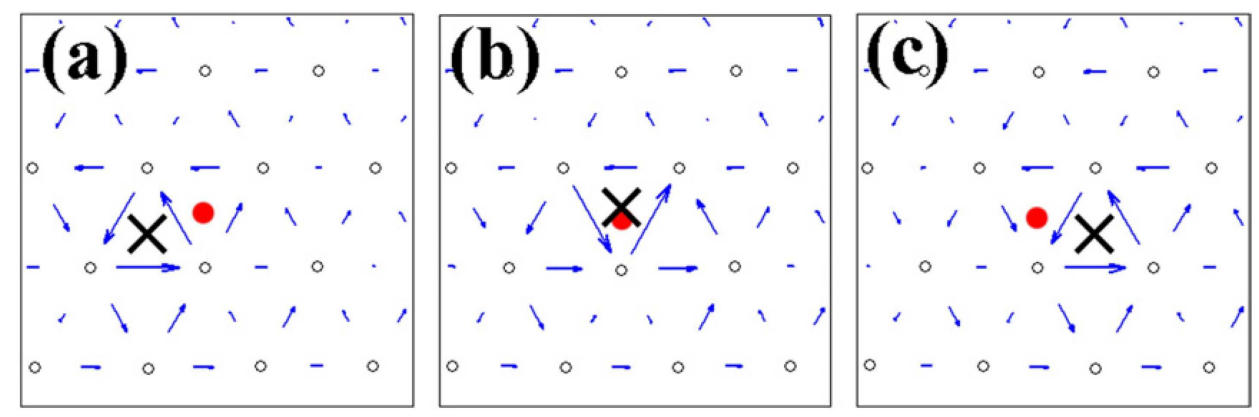

Figure 1.5 The differential displacement maps of the screw dislocation moving along the minimum energy path: (a) initial structure, (b) saddle point and (c) final structure. The dislocation core is represented by the cross and the position of the $\mathrm{H}$ impurity is indicated by a red dot. From reference [76].

To study the effect of $\mathrm{H}$ on dislocation mobility, the QM/MM method is employed to calculate the Peierls energy barrier which is the minimum energy that a straight dislocation has to overcome in order to move in a crystal. The screw dislocation is studied here because its mobility is much lower than that of the edge dislocation in $\mathrm{Fe}$, hence its mobility is more relevant to the overall mobility of a curved dislocation or a dislocation loop. The dislocation is placed at two adjacent "easy" positions as the initial and final state, as shown in Fig. 1.5(a) and (c) respectively. The minimal energy path is found to be in the $\{110\}$ plane, which is consistent with experiments [82]. The saddle point configuration (Fig. 1.5(b)) has a "none-split" core structure. The none-split core is very close to the "hard" core configuration while the split-core means that the dislocation spreads into two "easy" core configurations. The minimal energy path profile has a single-peak, i.e., the Peierls barrier. The Peierls energy barrier for the pure screw dislocation is $0.06 \mathrm{eV} / \mathrm{b}$, where $\mathrm{b}$ is the length of Burgers vector. In the presence of the $\mathrm{H}$ impurity, the Peierls barrier becomes $0.03 \mathrm{eV} / \mathrm{b}$ so that $\mathrm{H}$ can enhance the dislocation mobility considerably. Thus the HELP mechanism is also operating in Fe which is consistent with experimental observations. The reduction in the Peierls energy barriers can be explained from the $\gamma$-energy. With one $\mathrm{H}$ atom per $\langle 112\rangle \times \frac{3}{2}\langle 111\rangle$ cell $\left(49 \AA^{2}\right)$, we find that $\mathrm{H}$ can lower the $\gamma$-energy by 0.08 $\mathrm{J} / \mathrm{m}^{2}$. This is because in the presence of $\mathrm{H}$, the covalent Fe-Fe bonding across the slip plane is significantly disrupted and Fe-H ionic bonding is formed at its expense. Since the ionic Fe-H bonding across the slip plane resembles the formation of positively and negatively charged plates, the energy cost does not depend as sensitively to the shear as for the covalent Fe-Fe bonding. Hence the $\gamma$-energy in the presence of $\mathrm{H}$ is lower in Fe. 


\subsubsection{Hydrogen Assisted Cracking}

The presence of $\mathrm{H}$ is known to promote crack growth in a variety of technologically important materials [83]. A recent work on large-scale atomistic simulations of Al has suggested an interesting mechanism for ductile fracture, which depends on dislocation mobility and pinning behavior [84]. Based on EAM simulations, the authors have observed that the cleavage fracture can occur after emission of dislocations from the crack tip. The emitted dislocations can shield the crack tip, so that the macroscopic toughness exceeds the Griffith value, and hence the failure is a combination of both "brittle-like" and "ductile-like" phenomena. To fully address this problem, QCDFT that combines ab initio calculations for H-metal interactions near the crack tip and large-scale QC simulations for the rest of the system, is required. Here, we study $\mathrm{H}$-assisted cracking in $\mathrm{Al}$ which is at the heart of $\mathrm{H}$ embrittlement. If one understands the fundamental processes of $\mathrm{H}$ assisted cracking, it might be possible to establish connections between the various mechanisms and arrive at a comprehensive picture of $\mathrm{H}$ embrittlement.

Table 1.2 Summary of $K_{\mathrm{IC}}$ and $\Delta$ for eight crack configurations. Symbols in the configuration reflect the adsorbed $\mathrm{H}$ positions, e.g., $\mathrm{F}_{6}^{\mathrm{B}}$ means that there are six $\mathrm{H}$ atoms adsorbed on the bridge or B-site at the crack front-surface. $N_{\mathrm{H}}$ is the total number of adsorbed $\mathrm{H}$ atoms at the crack tip. From reference [85].

\begin{tabular}{ccccc}
\hline Case & Configuration & $N_{\mathrm{H}}$ & $K_{\mathrm{IC}}\left(\mathrm{eV} / \AA^{2.5}\right)$ & $\Delta(\AA)$ \\
\hline pure & pure & 0 & 0.28 & 0.16 \\
A & $\mathrm{U}_{2}^{\mathrm{B}}+\mathrm{D}_{2}^{\mathbf{B}}$ & 4 & 0.29 & 1.02 \\
B & $\mathrm{U}_{2}^{\mathbf{T}}+\mathrm{D}_{2}^{\mathbf{T}}$ & 4 & 0.28 & 0.21 \\
$\mathrm{C}$ & $\mathrm{F}_{6}^{\mathrm{B}}$ & 6 & 0.32 & 1.45 \\
$\mathrm{D}$ & $\mathrm{F}_{1}^{\mathbf{T}}$ & 1 & 0.31 & 6.1 \\
$\mathrm{E}$ & $\mathrm{F}_{5}^{\mathbf{T}}$ & 5 & 0.32 & 9.07 \\
$\mathrm{~F}$ & $\mathrm{~F}_{6}^{\mathrm{B}}+\mathrm{U}_{2}^{\mathrm{B}}$ & 8 & 0.30 & 1.35 \\
$\mathrm{G}$ & $\mathrm{F}_{6}^{\mathrm{B}}+\mathrm{U}_{2}^{\mathrm{B}}+\mathrm{D}_{2}^{\mathbf{B}}$ & 10 & 0.32 & 1.55 \\
$\mathrm{H}$ & $\mathrm{F}_{5}^{\mathbf{T}}+\mathrm{U}_{2}^{\mathbf{T}}+\mathrm{D}_{2}^{\mathbf{T}}$ & 9 & 0.30 & 8.42 \\
\hline & & & &
\end{tabular}

A semi-infinite crack (as shown in Fig. 1.3) in a single $\mathrm{Al}$ crystal with $\mathrm{H}$ atoms at the crack tip has been studied by using the QCDFT method [85]. Ab initio studies have showed that the Al-Al bond can be attacked by $\mathrm{H}$ with a significant charge transfer from the $\mathrm{Al}$ atoms to $\mathrm{H}[34,78,86]$. Hence, we consider two $\mathrm{H}$ adsorption sites at the crack tip in the QCDFT simulations: B site on the bridge center of an Al-Al bond, and $\mathbf{T}$ site right on top of an $\mathrm{Al}$ atom. The $\mathrm{H}$ atom at the $\mathbf{B}$-site forms an $\mathrm{Al}-\mathrm{H}-\mathrm{Al}$ bond whereas the $\mathrm{H}$ atom at the T-site forms an Al-H bond. At the crack tip, $\mathrm{H}$ atoms can be adsorbed on the upsurface (U), down-surface (D) and front-surface (F). To examine all these possibilities, we study eight different cases labeled from $(\mathrm{A})$ to $(\mathrm{H})$ as in Table 1.2. The primary quantities of interest here are the critical stress intensity for dislocation emission, $K_{\mathrm{IC}}$ and the crack opening at the stress intensity of $0.3 \mathrm{eV} / \AA^{2.5}, \Delta$. As shown in Table 1.2 , we find that $\mathrm{H}$-assist cracking occurs only when $\mathrm{H}$ atoms are adsorbed at the $\mathbf{T}$-site of the crack frontsurface; $\mathrm{H}$ concentration at $\sim 0.2 \mathrm{ML}$ would be sufficient to lead to crack growth as shown in case D. However, $\mathrm{H}$ atoms adsorbed on the side surfaces (both up- and down- surfaces) cannot yield crack growth. On the other hand, $\mathrm{H}$ atoms at the $\mathbf{T}$-site and $\mathbf{B}$-site on the crack front-surface could modify the pattern of dislocation nucleation from the crack tip. 

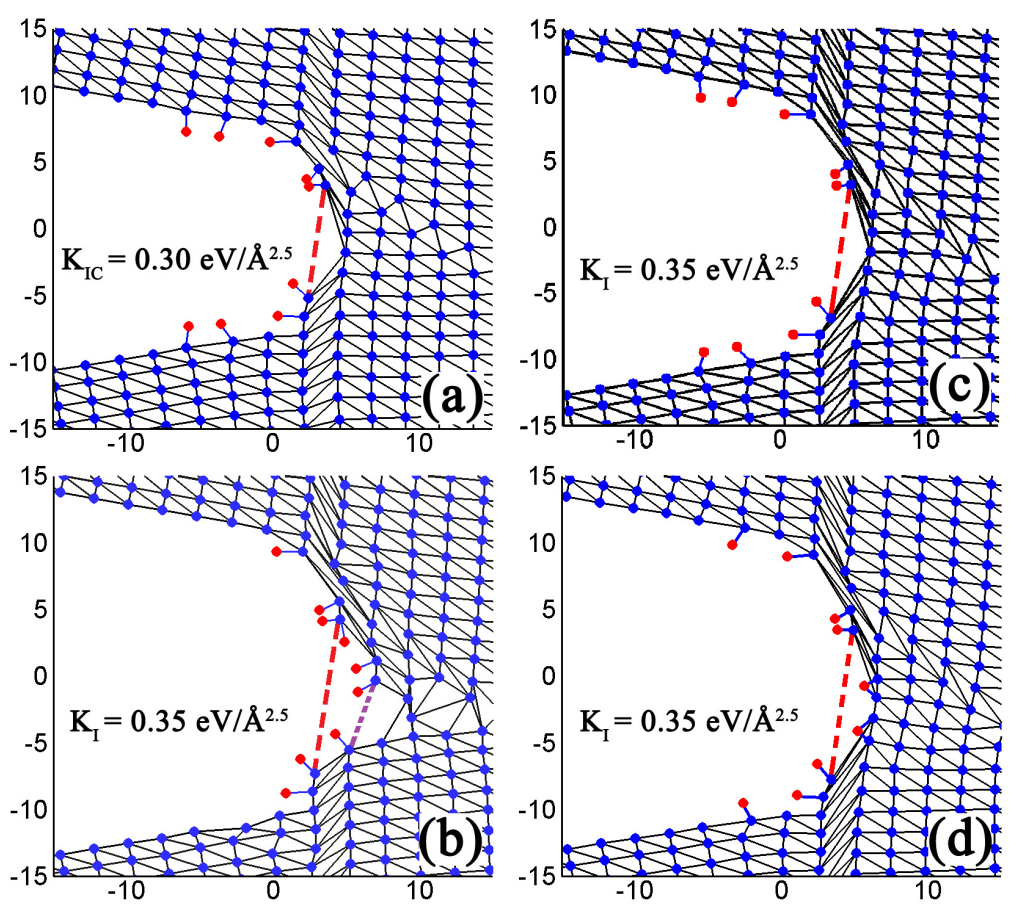

Figure 1.6 (a) Cleavage of the first atomic layer at the crack tip; the second atomic layer becomes the new front-surface subsequently. (b) Cleavage of the second atomic layer when $\mathrm{H}$ atoms are adsorbed on the $\mathbf{T}$-sites of the fresh front surface. No cleavage of the second atomic layer for (c) without $\mathrm{H}$ atoms on the fresh front-surface and (d) $\mathrm{H}$ atoms adsorbed on the $\mathbf{B}$-sites of the fresh frontsurface. The red and purple dotted lines represent the first and second atomic layers, respectively. From reference [85].

The preceding results suggest the following atomic processes of $\mathrm{H}$-assisted cracking. (1) At the crack front-surface, $\mathrm{H}$ atoms adsorbed on the $\mathbf{T}$-sites attack $\mathrm{Al}-\mathrm{Al}$ bonds which ultimately leads to the fracture of the first atomic layer as shown in Fig. 1.6A; the second atomic layer then becomes the new front-surface. (2) The $\mathrm{H}$ atoms at the original frontsurface and/or the side surfaces can diffuse to the fresh front-surface. The diffusion energy barrier is about $0.2 \mathrm{eV}$ which is similar to the $\mathrm{H}$ diffusion barrier on $\mathrm{Al}$ surfaces [87]. $\mathrm{H}$ adsorption and diffusion are key steps in $\mathrm{H}$ embrittlement. (3) With increasing loading level, $\mathrm{H}$ atoms on the $\mathbf{T}$-sites of the fresh front-surface continue attacking the Al-Al bonds at the second atomic layer as shown in Fig. 1.6B. Steps (2) and (3) repeat for a continuous crack growth. To confirm that only the $\mathrm{H}$ atoms on the T-sites can contribute to step (3), we study two contrasting cases with and without $\mathrm{H}$ atoms at the $\mathbf{B}$-sites of the fresh front-surface. At the stress intensity of $0.35 \mathrm{eV} / \AA^{2.5}$, the fracture of the second atomic layer occurs only when $\mathrm{H}$ atoms are adsorbed on the $\mathbf{T}$-sites as shown in Fig. 1.6B with a significant opening of $\Delta=3.9 \AA$ at the fresh front-surface. On the other hand, in the two contrasting cases, the crack does not grow as indicated in Fig. 1.6C and D.

To understand ductile-to-brittle transitions, Rice criterion in which the ratio between the surface energy (Griffith fracture energy) to the unstable stacking fault energy is often used [79]. The lower the ratio, the stronger the tendency for a brittle fracture; the higher the ratio, the more ductile the material behaves. While this picture may be overly simplistic 
for a quantitative analysis, it does give a useful insight for general trends and can even lead to predictions that have been verified experimentally [88]. We have used the Rice criterion to examine $\mathrm{H}$ embrittlement in $\mathrm{Mg}$ and find that the $\mathrm{H}$ impurities generally lower the ratio leading to the brittle fracture of $\mathrm{Mg}$ [77].

\subsubsection{Crucial Role of Vacancies}

Vacancies, being ubiquitously present in solids and having the ability to act as impurity traps, could play a central role in $\mathrm{H}$ embrittlement as hinted by some recent experiments. One set of experiments has established that $\mathrm{H}$ could induce superabundant vacancy formation in a number of metals, such as $\mathrm{Pd}, \mathrm{Ni}, \mathrm{Cr}$, etc. [89, 90]. The estimated vacancy concentration, $C_{\mathrm{v}}$, in these systems can reach a value as at $23 \%$ [90]. A conclusion drawn from these experiments is that $\mathrm{H}$ atoms, originally at bulk interstitials are trapped at vacancies in multiple numbers with rather high binding energies. The consequence of $\mathrm{H}$ trapping is that the formation energy of a vacancy defect is lowered by a significant amount. Such reduction in the vacancy formation energy could result in a drastic increase $\left(10^{7}\right.$ fold for $\mathrm{Fe}$ ) of equilibrium vacancy concentrations [91]. The superabundant vacancy formation in turn provides more trapping sites for $\mathrm{H}$ impurities, effectively increasing the apparent $\mathrm{H}$ solubility in metals by many orders of magnitude. For example, it was observed experimentally that about 1000 at. ppm of $\mathrm{H}$ atoms can enter $\mathrm{Al}$ accompanied by vacancy formation at the surface under aggressive $\mathrm{H}$ charging conditions, which should be contrasted with the equilibrium solubility of $\mathrm{H}$ in $\mathrm{Al}$ of about $10^{-5}$ at. $\mathrm{ppm}$ at room temperature where the experiments were carried out [92]; this is a staggering change of eight orders of magnitude in concentration. It was further observed that the $\mathrm{H}$-vacancy defects clustered and formed platelets lying on the $\{111\}$ planes, which directly lead to void formation or crack nucleation on the $\{111\}$ cleavage planes [92]. Because of the extremely low solubility of $\mathrm{H}$ in metals such as aluminum, experiments are usually difficult and results are dependent on $\mathrm{H}$ charging conditions; for such systems, first-principles calculations are particularly useful to complement experiments.

First-principles calculations show that the tetrahedral interstitial site for $\mathrm{H}$ atoms in bulk $\mathrm{Al}$ is slightly more favorable than the octahedral interstitial site by $0.07 \mathrm{eV}$. And the $\mathrm{H}$ atom prefers to occupy the vacancy site over the interstitial tetrahedral site in bulk by $0.40 \mathrm{eV}$ in excellent agreement with the experimental value of $0.52 \mathrm{eV}$ [1]. The lowest energy position for the $\mathrm{H}$ atom in the presence of a vacancy is not at the geometric center of the vacancy site, but rather at an off-center position close to a tetrahedral site adjacent to the vacancy site as shown in Fig. 1.7A. The $\mathrm{H}$ atom is negatively charged, and the kinetic energy of the $\mathrm{H}^{-1}$ electrons is lowered at the vacancy site where the conduction electron density is lower. At the same time, it is energetically favorable for the $\mathrm{H}^{-1}$ ion to sit off center of the vacancy, to minimize the Coulomb interaction energy with the nearby $\mathrm{Al}$ ions. Having established the stability of a single $\mathrm{H}$ atom at a single vacancy in $\mathrm{Al}$, the ensuing question is whether multiple $\mathrm{H}$ atoms, in particular, $\mathrm{H}_{2}$ molecules would be stable at this defect. This question is relevant to $\mathrm{H}_{2}$ bubble formation that gives rise to $\mathrm{H}$ embrittlement. The binding energy $E_{\mathrm{b}}$ of the $\mathrm{H}_{2}$ unit at a vacancy site is calculated as

$$
E_{\mathrm{b}}=E_{\mathrm{c}}\left(V_{\mathrm{Al}}+\mathrm{H}_{2}\right)+E_{\mathrm{c}}\left(V_{\mathrm{Al}}\right)-2 E_{\mathrm{c}}\left(V_{\mathrm{Al}} \mathrm{H}\right),
$$

where $E_{\mathrm{c}}\left(V_{\mathrm{Al}}+\mathrm{H}_{2}\right)$ is the cohesive energy of a system with an $\mathrm{H}_{2}$ unit at the center of the vacancy, $E_{\mathrm{c}}\left(V_{\mathrm{Al}}\right)$ is the cohesive energy of a system with a single vacancy in the absence of the $\mathrm{H}_{2}$ unit, and $E_{\mathrm{c}}\left(V_{\mathrm{Al}} \mathrm{H}\right)$ is the cohesive energy of a system with a single $\mathrm{H}$ atom at the vacancy (in the off-center tetrahedral site). This binding energy is $0.06 \mathrm{eV}$, 
simply stating that these $2 \mathrm{H}$ atoms would prefer to be trapped at two single vacancy sites individually rather than in the same vacancy site as a pair. One can conclude that if the single vacancy concentration $C_{\mathrm{v}}$ is greater than the $\mathrm{H}$ concentration $C_{\mathrm{H}}$, each vacancy in equilibrium should contain no more than $1 \mathrm{H}$ atom.

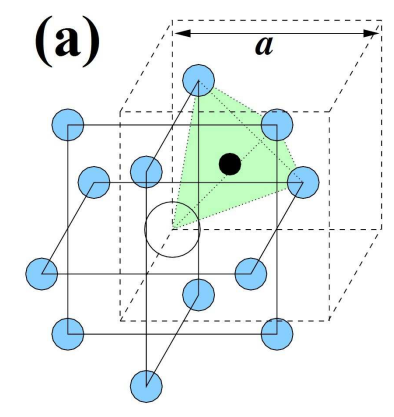

(c)
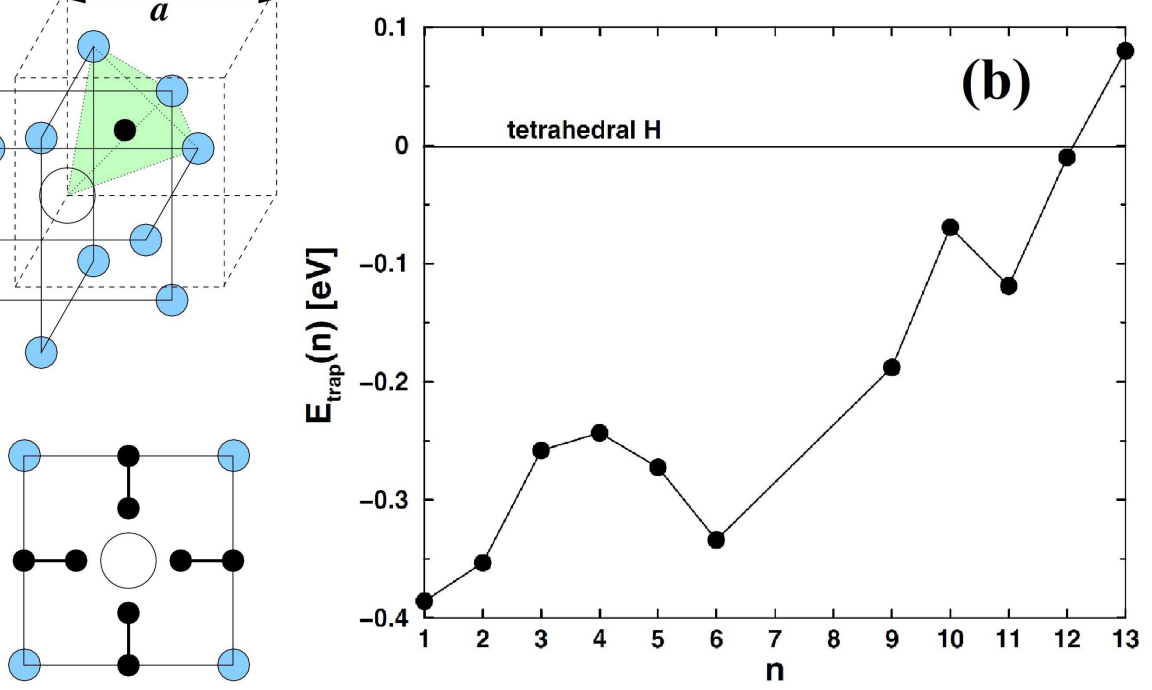

Figure 1.7 (a) The lowest energy site for a $\mathrm{H}$ atom (black circle) occupying in the vacancy. The vacancy is shown as a large open circle and its 12 nearest neighbors as smaller blue circles. (b) Trapping energy per $\mathrm{H}$ atom in $\mathrm{eV}$ as a function of the number of $\mathrm{H}$ atoms being trapped at a single vacancy site. (c) The arrangement of four of the six $\mathrm{H}_{2}$ molecules surrounding the vacancy on a [100] plane. From reference [93].

On the other hand, if $C_{\mathrm{H}}$ is greater than $C_{\mathrm{v}}$, the question arises as to where will the extra $\mathrm{H}$ atoms be situated, at interstitial or at vacancy sites? To answer this question, one can calculate the trapping energy $E_{\text {trap }}$ of multiple $\mathrm{H}$ atoms at a single vacancy site, which is defined as

$$
E_{\text {trap }}=\frac{1}{n}\left[E_{\mathrm{c}}\left(V_{\mathrm{Al}}+n \mathrm{H}\right)-E_{\mathrm{c}}\left(V_{\mathrm{Al}}\right)\right]-\left[E_{\mathrm{c}}^{0}(\mathrm{H})-E_{\mathrm{c}}^{0}\right]
$$

where $E_{\mathrm{c}}\left(V_{\mathrm{Al}}+n \mathrm{H}\right)$ is the cohesive energy of a system with $n \mathrm{H}$ atoms each situated at a single vacancy site, $E_{\mathrm{c}}^{0}(\mathrm{H})$ is the cohesive energy of bulk $\mathrm{Al}$ with a $\mathrm{H}$ atom at the tetrahedral interstitial site, and $E_{\mathrm{c}}^{0}$ is the cohesive energy of the ideal bulk without $\mathrm{H}$. A negative value for the trapping energy represents the energy gain when the $\mathrm{H}$ atoms are trapped at a single vacancy site relative to being dispersed at $n$ different tetrahedral interstitial sites. Here, the trapping energy was calculated to estimate the maximum number of $\mathrm{H}$ atoms trapped at a vacancy. An entire thermodynamic description of $\mathrm{H}$-vacancy interaction would depend on $\mathrm{H}$ chemical potential, temperature, pressure and other parameters. A more detailed analysis of such interaction has been put forwarded recently by Ismer et al. [95].

The results for $E_{\text {trap }}$ as a function of $n$ are summarized in Fig. 1.7B. It is energetically favorable for multiple $\mathrm{H}$ atoms to be trapped at a single vacancy site relative to being 
dispersed at interstitial sites as individual atoms. In fact, up to twelve $\mathrm{H}$ atoms can be trapped at a single vacancy in Al. The atomic arrangement of the $12 \mathrm{H}$ atoms trapped at a single vacancy is indicated in Fig. 1.7C. The ordered arrangement of the $\mathrm{H}$ atoms is necessary to minimize the electrostatic energy. Due to the trapping effect, vacancies can lead to a significant reduction in apparent lattice diffusivity of $\mathrm{H}$ in $\mathrm{Al}$, causing a strong composition dependence and non-Arrhenius behavior of the effective diffusion coefficient [94].

To check the possibility of $\mathrm{H}$-induced vacancy clustering, a number of relevant configurations have been examined including (i) two vacancies, each with $1 \mathrm{H}$ atom, forming a $\mathrm{NN}$ di-vacancy with $2 \mathrm{H}$ atoms trapped; (ii) $n$ vacancies, each with $2 \mathrm{H}$ atoms, forming a complex of NN multi-vacancies with $2 n \mathrm{H}$ atoms trapped, for $n=2$ and 3 . The results are summarized by using the notation of chemical reactions as

$$
\begin{gathered}
\text { (i) }: 2 V_{\mathrm{Al}} H \rightarrow\left(V_{\mathrm{Al}}\right)_{2} \mathrm{H}_{2}-0.21 \mathrm{eV}, \\
\text { (ii) }: n V_{\mathrm{Al}} \mathrm{H}_{2} \rightarrow\left(V_{\mathrm{Al}}\right)_{n} \mathrm{H}_{2 n}+n 0.29 \mathrm{eV}
\end{gathered}
$$

where the last number in each equation represents the reaction enthalpy. A positive value of enthalpy means the reaction is exothermic, that is, the process from left to right is energetically favorable. Consistent with the previous discussion, the reaction (i) is unfavorable (endothermic) because the effect of a single $\mathrm{H}$ atom on the covalent or metallic bonding of the NN Al atoms around the vacancy site is small and localized. On the other hand, reaction (ii) is favorable for $n=2$ and 3 , because the $\mathrm{H}_{2}$ units can attract more conduction electrons from the nearby $\mathrm{Al}$ atoms, weakening the bonding among the $\mathrm{NN} \mathrm{Al}$ atoms, which in turn drives the formation of multi-vacancies. The large energy gain in forming the tri-vacancy $(n=3)$ is of particular interest. First, it is consistent with the experimental observation that the single vacancy defects occupied by $\mathrm{H}$ atoms can coalesce to form platelets on $\{111\}$ planes of Al. Although the calculations primarily concern the formation of the tri-vacancy, it is likely that even larger vacancy clusters can also be formed based on the same mechanism. Second, these vacancy clusters can serve as embryos of cracks and microvoids with local $\mathrm{H}$ concentrations much higher than the average bulk value.

In general, the fracture surface is along the active slip planes where shear localization occurs. For fcc metals, the slip planes are the $\{111\}$ planes. In many cases, microvoids open up along these active slip planes in front of the crack tip; these microvoids can open and close in response to the local stress. Fracture occurs when these microcracks are joined to the crack tip, upon reaching the critical stress. The H-enriched microvoids may be created along the slip planes by the coalescence of vacancies with trapped $\mathrm{H}$. These microvoids can be formed only in the presence of $\mathrm{H}$, which produces an additional source of microcracks necessary for the $\mathrm{H}$ embrittlement. On the other hand, the apparent lattice mobility of $\mathrm{H}$ atoms is also enhanced since multiple $\mathrm{H}$ atoms may be trapped at a single vacancy. All these vacancy-based mechanisms contribute to the $\mathrm{H}$ embrittlement as they increase the rate of crack growth. Finally, the significant $H$ trapping at vacancies provides a scenario by which drastic increase of local $\mathrm{H}$ concentration may occur without improbable accumulation of $\mathrm{H}$ at bulk interstitial sites. This new feature resolves the longstanding problem of how a sufficiently high $\mathrm{H}$ concentration can be realized to induce $\mathrm{H}$ embrittlement in materials such as $\mathrm{Al}$, where the equilibrium $\mathrm{H}$ concentration in bulk is extremely low. 


\subsubsection{Hydrogen Diffusion}

$\mathrm{H}$ diffusion in metals plays an important role in $\mathrm{H}$ embrittlement. For example, it is observed experimentally that HELP occurs only when the thermal diffusion of $\mathrm{H}$ in the lattice is fast enough to follow the motion of dislocations [1]. In addition to their trapping of $\mathrm{H}$, dislocations and grain boundaries are believed to provide short-circuit paths for accelerated diffusion. Dislocations and grain boundaries could act as fast paths for diffusing atoms whose mobility can be orders of magnitude higher than in bulk diffusion and this phenomenon is often referred as "pipe diffusion". Furthermore, $\mathrm{H}$ diffusion at the crack tip under stress is central to $\mathrm{H}$-assisted cracking. Therefore, it is of great importance to determine $\mathrm{H}$ diffusion energy barriers in extended defects. To this end, we have employed $\mathrm{QM} / \mathrm{MM}$ approaches to calculate $\mathrm{H}$ diffusion energy barriers along dislocations and grain boundaries in $\mathrm{Fe}, \mathrm{Al}$, and $\mathrm{Mg}[53,76,77]$. Being important by themselves, these energy barriers could also be incorporated in kinetic Monte Carlo or other models for sequential multiscale modeling of $\mathrm{H}$ embrittlement in metals.
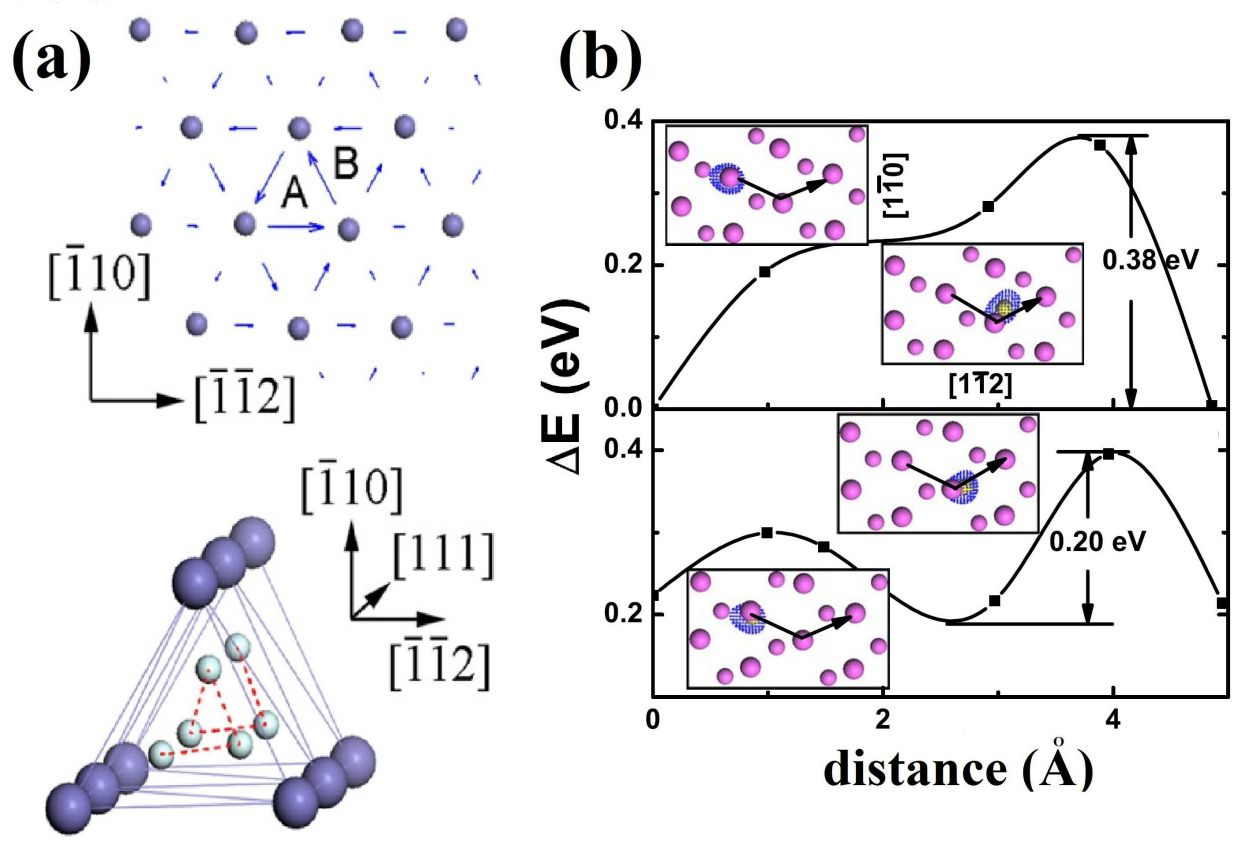

Figure 1.8 (a) $\mathrm{H}$ diffusion along the screw dislocation in Fe. Top panel: The differential displacement map of the screw dislocation. Bottom panel: The diffusion path represented by the red dashed curve passing through the tetrahedral sites along pipe A. (b) The energy profile for the $\mathrm{H}$ diffusion along partial core (top panel) and stacking fault ribbon (bottom panel). Inset: the schematic trajectory of the $\mathrm{H}$ atom along the diffusion path on the (111) plane. From references [76] and [53].

We have carried out QM/MM calculation to study $\mathrm{H}$ diffusion in bulk and along dislocations in $\alpha$-Fe. We find that the $\mathrm{H}$ diffusion energy barrier is $0.09 \mathrm{eV}$ in the bulk $\mathrm{Fe}$ between two adjacent bulk tetrahedral sites. Along a screw dislocation, $\mathrm{H}$ could diffuse between adjacent tetrahedral sites with two different diffusion paths: (A) along the dislocation center in which all tetrahedra are equally distorted and (B) along a path adjacent to 
the dislocation center in which the distortions are the not the same, as shown in Fig. 1.8A. The simulation results show that the diffusion energy barrier is $0.035 \mathrm{eV}$ along the path $\mathrm{A}$ and $0.063 \mathrm{eV}$ along the path B [76]; both values are smaller than that in the bulk. Similar $\mathrm{QM} / \mathrm{MM}$ calculations have been performed in $\mathrm{Al}$, and we find that the energy barriers are $0.26 \mathrm{eV}, 0.38 \mathrm{eV}$, and $0.20 \mathrm{eV}$ for $\mathrm{H}$ diffusion along grain boundaries, partial dislocation cores, and the stacking fault ribbon, respectively [53]. Along the same crystallographic directions, the corresponding diffusion barriers in the crystalline bulk lattice are $0.40 \mathrm{eV}$, $0.32 \mathrm{eV}$, and $0.32 \mathrm{eV}$, respectively. Based on the diffusion energy barriers, one can estimate the $\mathrm{H}$ diffusivity

$$
D=\nu l^{2} \exp \left[-\Delta E / k_{\mathrm{B}} T\right]
$$

where $\nu$ is the attempt frequency, $l$ is the hopping length, and $\Delta E$ is the diffusion energy barrier. At room temperature, there are two (one) orders of magnitude increase of diffusivity for $\mathrm{H}$ diffusion in $\mathrm{Al}(\mathrm{Fe})$ dislocations comparing to the bulk diffusivity. Therefore, dislocations in $\mathrm{Al}$ and $\mathrm{Fe}$ indeed can sever as fast pipes for $\mathrm{H}$ diffusion. The consequence of these results on $\mathrm{H}$ embrittlement is being pursued in our group.

However, we find that the basal dislocations of $\mathrm{Mg}$ are not easier paths for $\mathrm{H}$ diffusion [77]. In bulk Mg, the $\mathrm{H}$ diffusion consists of two types: (1) between the adjacent tetrahedral sites surrounded by octahedral sites; (2) between the octahedral site and its neighboring tetrahedral site. The long-range diffusion requires a combination of the two types. The energy barriers of case (1) and (2) are $0.04 \mathrm{eV}$ and $0.21 \mathrm{eV}$ respectively. The higher value $0.21 \mathrm{eV}$ is the actual energy barrier for the long-range diffusion. These values are consistent with experimental results [96, 97]. Along the partial dislocation core of $\mathrm{Mg}$, the QM/MM results show that the barriers slightly increase to $0.22 \mathrm{eV}$ and $0.26 \mathrm{eV}$ for the screw and edge dislocation respectively. And in the stacking fault ribbon the corresponding barriers become $0.25 \mathrm{eV}$ and $0.47 \mathrm{eV}$. Therefore, the energy barrier for $\mathrm{H}$ diffusion in $\mathrm{Mg}$ dislocations is actually greater than that in the crystalline lattice, in contrast to the cases in $\mathrm{Al}$ and $\mathrm{Fe}$. More investigations are desired to understand the ramifications of these results in $\mathrm{H}$ embrittlement of $\mathrm{Mg}$. Finally, we have also examined $\mathrm{H}$ diffusion at the crack tip of $\mathrm{Al}$ and find that $\mathrm{H}$ can diffuse with relative ease on the crack surfaces with lower energy barriers than those in the bulk; the $\mathrm{H}$ atoms on the fracture surfaces can even recombine into $\mathrm{H}_{2}$ molecules before re-dissociation at the fresh crack front surface to attack Al-Al bonds [85].

\subsection{Summary and Outlook}

Based on the experimental observations and the simulation results, we can tentatively identify the following key processes leading to $\mathrm{H}$ embrittlement of metals: (a) Application of external stress produces local concentration of tensile stress in the vicinity of cracks, which attracts $\mathrm{H}$ since $\mathrm{H}$ prefers to stay in slightly enlarged interstitial sites. $\mathrm{H}$ impurities can also be adsorbed at the crack surfaces near the crack tip; the relative ease of $\mathrm{H}$ diffusion on the crack surfaces may provide a rapid means of transporting $\mathrm{H}$ to the fresh crack front. (b) $\mathrm{H}$ can also segregate at vacancies inside the material which may lead to microvoids with high local $\mathrm{H}$ concentrations; the microvoids could merge to form cracks under stress. (c) The segregated $\mathrm{H}$ impurities at the crack tip can facilitate dislocation generation and deformation twining and enhance dislocation mobility, which will lead to extensive plastic deformation in front of the crack. The highly deformed region ahead of the crack tip becomes the "weakest link" where fracture takes place. (d) The highly deformed and disordered region along with the localization of slip due to the inhibition of cross-slip, allows 
the crack to propagate at lower stress levels, prior to general yielding away from the crack tip. These processes are not likely to be the only mechanism operating in $\mathrm{H}$ embrittlement [70], but are the most relevant processes in line with our multiscale modeling results on aluminum. The similar mechanism could be operative in other metals as well, but it should be further validated to be certain.

Multiscale modeling will continue to be important for understanding $\mathrm{H}$ embrittlement phenomena in metals. One area of particular importance and challenge is to model stress corrosion cracking under realistic conditions and in aqueous environment. The molecular structure of solid-liquid interface, $\mathrm{pH}$ of electrolyte, surface charge, field and electrochemical potential are all relevant parameters that need to be included in the modeling. Ab initio methods for modeling the charged solid/liquid interface at the atomic level are starting to appear [98, 99, 100, 101]. An interesting future direction is to incorporate this type of $a b$ initio modeling into QCDFT framework so that the chemo-mechanical and electrochemical processes important to the stress corrosion cracking can be captured.

We acknowledge the collaborators who have contributed to our understanding of $\mathrm{H}$ embrittlement in metals and the development of the multiscale methodologies presented in this chapter. The related research was supported primarily by NSF-PREM program, DoESciDAC program and the Office of Naval Research (ONR). We are particularly grateful to Kenny Lipkowitz for his encouragement and support.

\section{REFERENCES}

1. Myers SM, Baskes MI, Birnbaum HK, Corbett JW, DeLeo GG, Estreicher SK, Haller EE, Jena P, Johnson NM, Kirchheim R, Pearton SJ, Stavola MJ. Hydrogen interactions with defects in crystalline solids. Rev. Mod. Phys. 1992;64:559-617.

2. Oriani RA. Hydrogen embrittlement of steels. Ann. Rev. Mater. Sci. 1978;8:327-357.

3. Westlake DG. A generalized model for hydrogen embrittlement. Trans. ASM 1969;62:10001006.

4. Gahr S, Grossbeck ML, Birnbaum HK. Hydrogen embrittlement of Nb I - macroscopic behavior at low temperatures. Acta Metall. 1977;25:125-134.

5. Shi D, Robertson IM, Birnbaum HK. Hydrogen embrittlement of $\alpha$-titanium: in situ TEM studies. Acta Metall. 1988;36:111-124.

6. Beachem CD. A new model for hydrogen-assisted cracking (hydrogen embrittlement). Metall. Trans. 1972;3:437-451.

7. Sofronis P, Robertson IM. TEM observations and micromechanical/continuum models for the effect of hydrogen on the mechanical behavior of metals. Philos. Mag. A 2002;82:3405-3413.

8. Eastman J, Matsumoto T, Narita N, Heubaum N, Birnbaum HK. Hydrogen effects in nickel embrittlement or enhanced ductility? In: Bernstein IM, Thompson AW, editors. Hydrogen in metals. New York: AIME;1981. p 397-399.

9. Matsumoto T, Eastman J, Birnbaum HK. Direct observations of enhanced dislocation mobility due to hydrogen. Scripta Metall. 1981;15:1033-1037.

10. Oriani RA, Josephic PH. Equilibrium aspects of hydrogen-induced cracking of steels. Acta Metall, 1974;22:1065-1074.

11. Oriani RA, Josephic PH. Equilibrium and kinetic studies of the hydrogen-assisted cracking of steels. Acta Metall, 1977;25:979-988.

12. Steigerwald EA, Schaller FW, Troiano AR. The role of stress in hydrogen induced delayed failure. Trans. Metall Soc. AIME, 1960;218:832-841. 
13. Phillips R. Crystals, defects and microstructures - modeling across scales. Cambridge(UK): Cambridge University Press; 2001.

14. Lu G, Kaxiras E. Overview of Multiscale Simulations of Materials. In: Rieth M, Schommers W, editors. Handbook of Theoretical and Computational Nanotechnology. Stevenson Ranch: American Scientific; 2004. Chap. 22.

15. Kaxiras E, Yip S. Modeling and simulation of solids. Curr. Opin. Solid State Mater. Sci. 1998;3:523-525 and accompanying articles.

16. Diaz de la Rubia T, Bulatov VV. Materials research by means of multiscale computer simulation. MRS Bull. 2001;26:169-175 and accompanying articles.

17. Yip S. Synergistic science. Nature Mater. 2003;2:3-5.

18. Hohenberg P, Kohn W. Inhomogeneous electron gas. Phys. Rev. 1964;136:B864-B871.

19. Kohn W, Sham LJ. Self-consistent equations including exchange and correlation effects. Phys. Rev. 1965;140:A1133-A1138.

20. Abraham FF, Walkup R, Gao H, Duchaineau M, de la Rubia T, Seager M. Simulating materials failure by using up to one billion atoms and the world's fastest computer: work-hardening. Proc. Natl. Acad. Sci. USA, 2002;99:5777-5782.

21. Hughes TJR. The finite element method. Englewood Cliffs(NJ): Prentice-Hall; 1987.

22. Peierls R. The size of a dislocation. Proc. Phys. Soc. London 1940;52:34-37.

23. Nabarro FRN. Dislocations in a simple cubic lattice. Proc. Phys. Soc. London 1947;59:256-272.

24. Joos B, Ren Q, and Duesbery MS. Peierls-Nabarro model of dislocations in silicon with generalized stacking-fault restoring forces. Phys. Rev. B 1994;50:5890-5898.

25. Joos B, Duesbery MS. The Peierls Stress of Dislocations: An Analytic Formula. Phys. Rev. Lett. 1997;78:266-269.

26. Juan Y, and Kaxiras E. Generalized stacking fault energy surfaces and dislocation properties of silicon: a first-principles theoretical study. Philos. Mag. A 1996;74:1367-1384.

27. Hartford J, von Sydow B, Wahnstrom G, Lundqvist BI. Peierls barriers and stresses for edge dislocations in Pd and Al calculated from first principles. Phys. Rev. B 1998;58:2487-2496.

28. von Sydow B, Hartford J, Washnstrom G. Atomistic simulations and Peierls-Nabarro analysis of the Shockley partial dislocations in palladium. Comput. Mater. Sci. 1999;15:367-379.

29. Medvedeva NI, Mryasov ON, Gornostyrev YN, Novikov DL, Freeman AJ. First-principles totalenergy calculations for planar shear and cleavage decohesion processes in B2-ordered $\mathrm{NiAl}$ and FeAl. Phys. Rev. B 1996;54:13506-13514.

30. Mryasov ON, Gornostyrev YN, Freeman AJ. Generalized stacking-fault energetics and dislocation properties: Compact versus spread unit-dislocation structures in TiAl and CuAu. Phys. Rev. B 1998;58:11927-11932.

31. Bulatov VV, Kaxiras E. Semidiscrete Variational Peierls Framework for Dislocation Core Properties. Phys. Rev. Lett. 1997;78:4221-4224.

32. Lu, G, The Peierls-Nabarro Model of Dislocations: A Venerable Theory And Its Current Development. S. Yip (ed.), Handbook of Materials Modeling. Volume I: Methods and Models, 2005 Springer (Netherlands).

33. Lu G, Kioussis N, Bulatov VV, Kaxiras E. Generalized-stacking-fault energy surface and dislocation properties of aluminum. Phys. Rev. B 2000;62:3099-3108.

34. Lu G, Zhang Q, Kioussis N, Kaxiras E. Hydrogen-Enhanced Local Plasticity in Aluminum: An Ab Initio Study. Phys. Rev. Lett. 2001;87:095501.

35. Lu G, Kaxiras E. Can Vacancies Lubricate Dislocation Motion in Aluminum? Phys. Rev. Lett. 2002;89:105501. 
36. Lu G, Bulatov VV, Kioussis N. Dislocation constriction and cross-slip: An ab initio study. Phys. Rev. B 2002;66:144103.

37. Vitek V. Intrinsic stacking faults in body-centered cubic crystals. Philos. Mag. 1968;18:773-786.

38. Eshelby JD. Edge Dislocations in Anisotropic Materials. Philos. Mag. 1949;40:903-912.

39. Hirth JP, Lothe J. Theory of Dislocations. 2nd ed. New York:Wiley; 1992.

40. Bernstein N, Kermode JR, Csanyi G. Hybrid atomistic simulation methods for materials systems. Rep. Prog. Phys. 2009;72:026501.

41. Lin H, Truhlar DG. QM/MM: what have we learned, where are we, and where do we go from here? Theor. Chem. Acc. 2007;117:185-199.

42. Zhang X, Zhao Y, Lu G. Recent development in quantum mechanics/molecular mechanics modeling for materials. Int. J. Multiscale Comput. Eng. 2012;10:65-82.

43. Antes I, Thiel W. On the treatment of link atoms in hybrid methods. In: Gao J, Thompson MA, editors. Hybrid Quantum Mechanical and Molecular Mechanical Methods Proc. ACS Symp. Ser. Washington DC: ACS; 1998. 712:50-65.

44. Gao J, Truhlar DG. Quantum mechanical methods for enzyme kinetics. Annu. Rev. Phys. Chem. 2002;53:467-505.

45. Zhang X, Lu G, Curtin WA. Multiscale quantum/atomistic coupling using constrained density functional theory. Phys. Rev. B 2013;87:054113.

46. Huang P, Carter EA. Advances in Correlated Electronic Structure Methods for Solids, Surfaces, and Nanostructures. Annu. Rev. Phys. Chem. 2008;59:261-290.

47. Nair AK, Warner DH, Hennig RG, Curtin WA. Coupling quantum and continuum scales to predict crack tip dislocation nucleation. Scr. Mater. 2010;63:1212-1215.

48. Leyson GP, Curtin WA, Hector LG Jr, Woodward CF. Quantitative prediction of solute strengthening in aluminium alloys. Nature Mater. 2010;9:750-755.

49. Woodward C, Rao SI. Flexible Ab Initio Boundary Conditions: Simulating Isolated Dislocations in bcc Mo and Ta. Phys. Rev. Lett. 2002;88:216402.

50. Choly N, Lu G, E W, Kaxiras E. Multiscale simulations in simple metals: A density-functionalbased methodology. Phys. Rev. B 2005;71:094101.

51. Zhang X, Lu G. Quantum mechanics/molecular mechanics methodology for metals based on orbital-free density functional theory. Phys. Rev. B 2007;76:245111.

52. Zhang X, Wang CY, Lu G. Electronic structure analysis of self-consistent embedding theory for quantum/molecular mechanics simulations. Phys. Rev. B 2008;78:235119.

53. Zhang X, Peng Q, Lu G. Self-consistent embedding quantum mechanics/molecular mechanics method with applications to metals. Phys. Rev. B 2010;82:134120.

54. Daw MS, Baskes MI. Embedded-Atom method: Derivation and application to impurities, surfaces, and other defects in metals. Phys. Rev. B 1984;29:6443-6453.

55. Zhang X, Lu G. Quantum mechanics/molecular mechanics methodology based on constrained density functional theory. 2012; submitted.

56. Garcia Gonzalez P, Alvarellos JE, Chacon E. Nonlocal kinetic-energy-density functionals. Phys. Rev. B 1996;53:9509-9512.

57. Wang LW, Teter MP. Kinetic-energy functional of the electron-density. Phys. Rev. B 1992;45:13196-13220.

58. Wang YA, Govind N, Carter EA. Orbital-free kinetic-energy density functionals with a densitydependent kernel. Phys. Rev. B 1999;60:16350-16358.

59. Liu Y, Lu G, Chen ZZ, Kioussis N. An improved QM/MM approach for metals. Modelling Simul. Mater. Sci. Eng. 2007;15:275-284. 
60. Tadmor EB, Ortiz M, Phillips R. Quasi-continuum analysis of defects in solids. Philos. Mag. A 1996;73:1529-1563.

61. Lu G, Tadmor EB, Kaxiras E. From electrons to finite elements: a concurrent multiscale approach for metals. Phys. Rev. B 2006;73:024108.

62. Peng Q, Zhang X, Hung L, Carter EA, Lu G. Quantum simulation of materials at micron scales and beyond. Phys. Rev. B 2008;78:054118.

63. Peng Q, Lu G. A comparative study of fracture in Al: Quantum mechanical vs. empirical atomistic description. J. Mech. Phys. Solids 2011;59:775-786.

64. Birnbaum HK. Mechanism of hydrogen related fracture of metals. In: Moody NR, Thompson AW, editors. Hydrogen Effects on Material Behavior. Warrendale: TMS; 1990. p 629-660.

65. Tabata T, Birnbaum HK. Direct observations of the effect of hydrogen on the behavior of dislocations in iron. Scripta Metall. 1983;17:947-950.

66. Tabata T, Birnbaum HK. Direct observations of hydrogen enhanced crack propagation in iron. Scripta Metall. 1984;18:231-236.

67. Robertson IM, Birnbaum HK. An HVEM study of hydrogen effects on the deformation and fracture of nickel. Acta Metall. 1986;34:353-366.

68. Bond GM, Robertson IM, Birnbaum HK. The influence of hydrogen on deformation and fracture processes in high-strength aluminum alloys. Acta Metall. 1987;35:2289-2296.

69. Bond GM, Robertson IM, and Birnbaum HK. Effects of hydrogen on deformation and fracture processes in high-purity aluminum. Acta Metall. 1988;36:2193-2197.

70. Birnbaum HK, Sofronis P. Hydrogen-enhanced localized plasticity - a mechanism for hydrogenrelated fracture. Mater. Sci. Eng. A 1994;176:191-202.

71. Ulmer DG, Altstetter CJ. Hydrogen-induced strain localization and failure of austenitic stainless-steels at high hydrogen concentrations. Acta Metall. Mater. 1991;39:1237-1248.

72. Tang X, Thompson AW. Hydrogen effects on slip character and ductility in Ni-Co alloys. Mater. Sci. Eng. A 1994;186:113-119.

73. Walston WS, Bernstein IM, Thompson AW. The effect of internal hydrogen on a single-crystal nickel-base superalloy. Metall. Trans. A 1992;23:1313-1322.

74. He J, Fukuyama S, Yokogawa K, Kimura A. Effect of hydrogen on deformation structure of inconel-718. Mater. Trans. JIM 1994;35:689-694.

75. McInteer WA, Thompson AW, Bernstein IM. The effect of hydrogen on the slip character of nickel. Acta Metall. 1980;28:887-894.

76. Zhao Y, Lu G. QM/MM study of dislocation-hydrogen/helium interactions in $\alpha$-Fe. Modelling Simul. Mater. Sci. Eng. 2011;19:065004.

77. Zhao Y, Lu G. H in Mg: energetics of decohesion, slip, twinning and diffusion. 2012; submitted.

78. Lu G, Orlikowski D, Park I, Politano O, Kaxiras E. Energetics of hydrogen impurities in aluminum and their effect on mechanical properties. Phys. Rev. B 2002;65:064102.

79. Rice JR. Dislocation nucleation from a crack tip: an analysis based on the Peierls concept. J. Mech. Phys. Solids 1992;40:239-271.

80. Daw MS, Baskes MI. Application of the embedded atom method to hydrogen embrittlement. In: Latanision RM, R. Jones R, editors. Chemistry and Physics of Fracture. Netherlands: Martinus Nijhoff Publishers; 1987. p 196-218.

81. Sofronis P, Birnbaum HK. Mechanics of the Hydrogen-Dislocation-Impurity Interactions: Part I-Increasing Shear Modulus. J. Mech. Phys. Solids 1995;43:49-90.

82. Spitzig WA, Keh AS. The effect of orientation and temperature on the plastic flow properties of iron single crystals. Acta. Metall. 1970;18:611-622. 
83. Lynch SP. Metallographic and Fractographic techniques for characterising and understanding hydrogen-assisted cracking of metals. In: Gangloff R, Somerday B, editors. Gaseous hydrogen embrittlement of materials in energy technologies. Cambridge: Woodhead; 2012. p 274-346.

84. Farkas D, Duranduru M, Curtin WA, Ribbens C. Multiple-dislocation emission from the crack tip in the ductile fracture of Al. Philos. Mag. A 2001;81:1241-1255.

85. Sun Y, Peng Q, Lu G. Hydrogen assisted cracking: a QCDFT study of Aluminum crack-tip. 2012; submitted.

86. Apostol $\mathrm{F}$ and Mishin $\mathrm{Y}$. Hydrogen effect on shearing and cleavage of $\mathrm{Al}$ : A first-principles study. Phys. Rev. B 2011;84:104103.

87. Gunnarsson O, Hjelmberg H, Lundqvist BI. Binding energies for different adsorption sites of hydrogen on simple metals. Phys. Rev. Lett. 1976;37:292-295.

88. Waghmare UV, Kaxiras E, and Duesbery MS. Modeling Brittle and Ductile Behavior of Solids from First-Principles Calculations. Phys. Status Solidi B 2000;217:545-564.

89. Fukai Y, Okuma N. Formation of Superabundant Vacancies in Pd Hydride under High Hydrogen Pressures. Phys. Rev. Lett. 1994;73:1640-1643.

90. Fukai Y. Superabundant Vacancies Formed in Metal Hydrogen Alloys. Phys. Scr. 2003;T103:1114.

91. Tateyama Y, Ohno T. Stability and clusterization of hydrogen-vacancy complexes in Fe: An ab initio study. Phys. Rev. B 2003;67:174105.

92. Birnbaum HK, Buckley C, Zeides F, Sirois E, Rozenak P, Spooner S, Lin JS. Hydrogen in aluminum. J. Alloy. Compd. 1997;253-254:260-264.

93. Lu G, Kaxiras E. Hydrogen Embrittlement of Aluminum: The Crucial Role of Vacancies. Phys. Rev. Lett. 2005;94:155501.

94. Gunaydin H, Barabash SV, Houk KN, Ozolins V. First-Principles Theory of Hydrogen Diffusion in Aluminum. Phys. Rev. Lett. 2008;101:075901.

95. Ismer L, Park MS, Janotti A, Van de Walle CG. Interactions between hydrogen impurities and vacancies in $\mathrm{Mg}$ and $\mathrm{Al}$ : A comparative analysis based on density functional theory. Phys. Rev. B 2009;80:184110.

96. Tsuru T, Latanision RM. Grain boundary transport of hydrogen in nickel. Scr. Metall. 1982;16:575-578.

97. Nishimura C, Komaki M, Amano M. Hydrogen permeation through magnesium. J. Alloys Compd. 1999;293-295:329-333.

98. Taylor CD, Sally A. Wasileski SA, Filhol JS, Neurock M. First principles reaction modeling of the electrochemical interface: Consideration and calculation of a tunable surface potential from atomic and electronic structure. Phys. Rev. B 2006;73:165402.

99. Filhol JS, Neurock M. Elucidation of the Electrochemical Activation of Water over Pd by First Principles. Angew. Chem. Int. Ed. 2006;45:402-406.

100. Schnur S, and Grob A. Properties of metal-water interfaces studied from first principles. New J. Phys. 2009;11:125003.

101. Rossmeisl J, Skulason E, Bjorketun ME, Tripkovic V, Norskov JK. Modeling the electrified solid-liquid interface. Chem. Phys. Lett. 2008;466:68-71. 